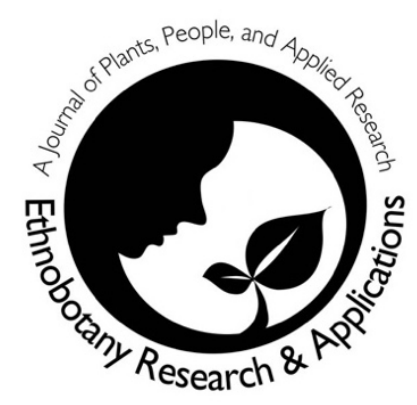

\title{
Ethnobotanical use and conservation assessment of medicinal plants sold in markets of Burkina Faso
}

\author{
Lassané Ouédraogo, Josef Endl, Pierre Alexandre Eric \\ Djifaby Sombié, Hanno Schaefer and Martin \\ Kiendrebeogo
}

\section{Research}

\begin{abstract}
Background: A comprehensive survey on the trade and use of medicinal plants in Burkina Faso was carried out with the aim of identifying the most relevant species and their conservation status.
\end{abstract}

Methods: Interviews of 30 medicinal plant traders in six major cities was carried out and the data on medicinal plant species were recorded using a structured questionnaire.

Results: A total of 104 medicinal plant species, belonging to 39 families and 81 genera were reported to treat various health problems. Most of the medicinal plant species found in the markets were used for gastrointestinal diseases (48\%), e.g., Pteleopsis suberosa (Combretaceae) and Bauhinia rufescens (Fabaceae). The combination of fidelity level and preference ranking identified Gardenia sokotensis, Combretum micranthum and Maytensus senegalensis as particularly important species for treatment of malaria. Four species found in local markets are listed as vulnerable in the IUCN red list of threatened species (Afzelia africana, Vitellaria paradoxa, Khaya senegalensis and Tapinanthus globiferus). For many species that are traded in large quantities sufficient data for precise assessment of their conservation status do not yet exist. Some species such as Securidaca longipedunculata and Zanthoxylum zanthoxyloides are already rare in nature and sold at high price on the stalls.

Conclusions: There is an urgent need to collect distribution data for the medicinal plant species of Burkina Faso and design conservation plans in order to preserve their natural populations.
Keywords: Ethnobotanical survey; Medicinal plants; Conservation status; Traditional knowledge; Diseases; Burkina Faso

\section{Correspondence \\ Lassané Ouédraogo ${ }^{1 *}$, Josef Endl², Pierre Alexandre Eric Djifaby Sombié ${ }^{1}$, Hanno Schaefer $^{3}$ and Martin Kiendrebeogo ${ }^{4}$ \\ ${ }^{1}$ Centre National de Recherche Scientifique et Technologique (CNRST), Institut de l'Environnement et de Recherches Agricoles, 03 BP 7047 Ouagadougou 03, Burkina Faso, email: lassanouedraogo@yahoo.fr ; ericsombie@yahoo.fr 2Ulmenstr.12, 82362 Weilheim, Germany, email: josef.endl@t-online.de \\ ${ }^{3}$ Plant Biodiversity Research, Technical University of Munich (TUM), Emil-Ramann Strasse 2, D-85354 Freising, Germany: email: hanno.schaefer@tum.de ${ }^{4}$ Laboratoire de Biochimie \& Chimie Appliquées, Université Joseph Ki-Zerbo, 03 BP 7021 Ouagadougou 03, Burkina Faso, email: martinkiendrebeogo@yahoo.co.uk \\ *Corresponding Author: lassanouedraogo@yahoo.fr \\ Ethnobotany Research \& Applications \\ 20:39 (2020)}

\section{Background}

Traditional medicine has played a key role in many developing countries and continues to do so (World Health Organization, WHO 2004). In this area, it is estimated that more than $80 \%$ of the people rely on plants for their primary healthcare requirements (Senthilkumar et al. 2013). 
In West African countries, particularly in Burkina Faso, traditional medicine and pharmacopoeia remain the main source of primary health care for an estimated $70 \%$ of the population (Zerbo et al. 2011). The main reasons for this dependence on herbal medicine are its therapeutic efficacy, accessibility and low costs compared to modern medicine and that it is deeply intertwined with traditional beliefs (Benoît 2008).

But the increased use of herbal medicines has also negative consequences. It propels safety concerns to both national health authorities and the general public, mostly due to misidentification, substitution of rare species with cheaper plant material from species which are more readily available and infection of herbs with fungi (Kool et al. 2012). In addition, it could negatively impact natural plant resources when exploitation is performed in an unsustainable way (WHO 2004).

Meticulous documentation, sustainable use and conservation efforts are therefore essential. Traditional medicine practitioners are officially recognized by the authorities of Burkina Faso in order to regulate their activities in collecting and selling medicinal plants ("Assemblée Nationale", AN 1994). These regulations are recorded in two national codes of Burkina Faso. In the Public Health Code recipes and use of medicinal plants are described (AN 1994). In the Forest Code traditional use rights are recognized for the benefit of local residents, allowing picking of fruits and harvesting of medicinal plants (AN 1997). Most of the medicinal plants are sold in urban markets (Cunningham 2014) and market surveys have become an efficient way of acquiring data on local consumption and conservation status of medicinal plant species (Idu et al. 2010).

It seems necessary to inform on the composition of medicinal plants traded in Burkina Faso, the quantities sold, the provenance of the plant material and the indigenous uses for disease treatment. Documentation of traditional uses of medicinal plants sold in the markets is important for several reasons. The identification of local names, scientific names and indigenous uses of plants not only preserves indigenous knowledge but also contributes to future research in order to improve the safety and efficacy of medicinal plants in the treatment of various ailments (Tugume et al. 2016). This will facilitate the integration of herbal medicine into the main health care system. In addition, this knowledge will support the conservation of cultural traditions and help to preserve the diversity of wild plant resources.

The knowledge on traditional medicinal uses of plants is disappearing at an alarming rate in Burkina Faso mainly because this information is only orally transmitted to the younger generation. Many of these custodians die before passing on the information to the next generations. The importance of saving this traditional knowledge is convincingly demonstrated by the fact that an estimated $25 \%$ of prescription drugs and $11 \%$ of drugs considered essential by $\mathrm{WHO}$ are based on active compounds first isolated from wild plants (Maroyi et al. 2011). Thus, a thorough knowledge of traditionally used medicinal plants could help to fully exploit their potential for discovering new sources of drugs and promote the sustainable use of these natural resources in Burkina Faso.

Therefore, we conducted this study on medicinal plant trade in the main local markets in six major cities (Ouagadougou, Fada, Dédougou, Léo, Ouahigouya, and Bobo-Dioulasso) of Burkina Faso. The study aims to deliver a comprehensive list of the medicinal plants together with their suggested applications. To assess the conservation status of each species, the provenance and estimated quantities of plant material offered by the sellers was documented and compared to International Union for Conservation of Nature (IUCN) red list status. Finally, we provide suggestions how to avoid overexploitation of selected species in order to preserve them for the benefit of future generations.

\section{Materials and Methods}

\section{Study sites}

The study focused on 18 markets of six cities in different climatic and vegetation zones of Burkina Faso. Selected cities for the survey were Ouagadougou, Ouahigouya, Fada N'Gourma, Dédougou, Bobo Dioulasso, and Léo (Figure 1). The target cities were chosen randomly in the four agroecological zones defined by Fontès and Guinko (2012). The three biggest markets of each city were selected as study sites. Ouagadougou and Ouahigouya are located in the Sahelian zone dominated by shrub savanna with annual rainfall less than $600 \mathrm{~mm}$. Dédougou and Fada N'Gourma belong to the northern Sudanese zone of the country where woody and shrubby savannas dominate and rainfall ranges from $600-900 \mathrm{~mm}$. Bobo-Dioulasso and Léo are located in the southern Sudanese zone with open forests and islands of dense dry forest with rainfall up to $900 \mathrm{~mm}$ (MAHRH 2007). 


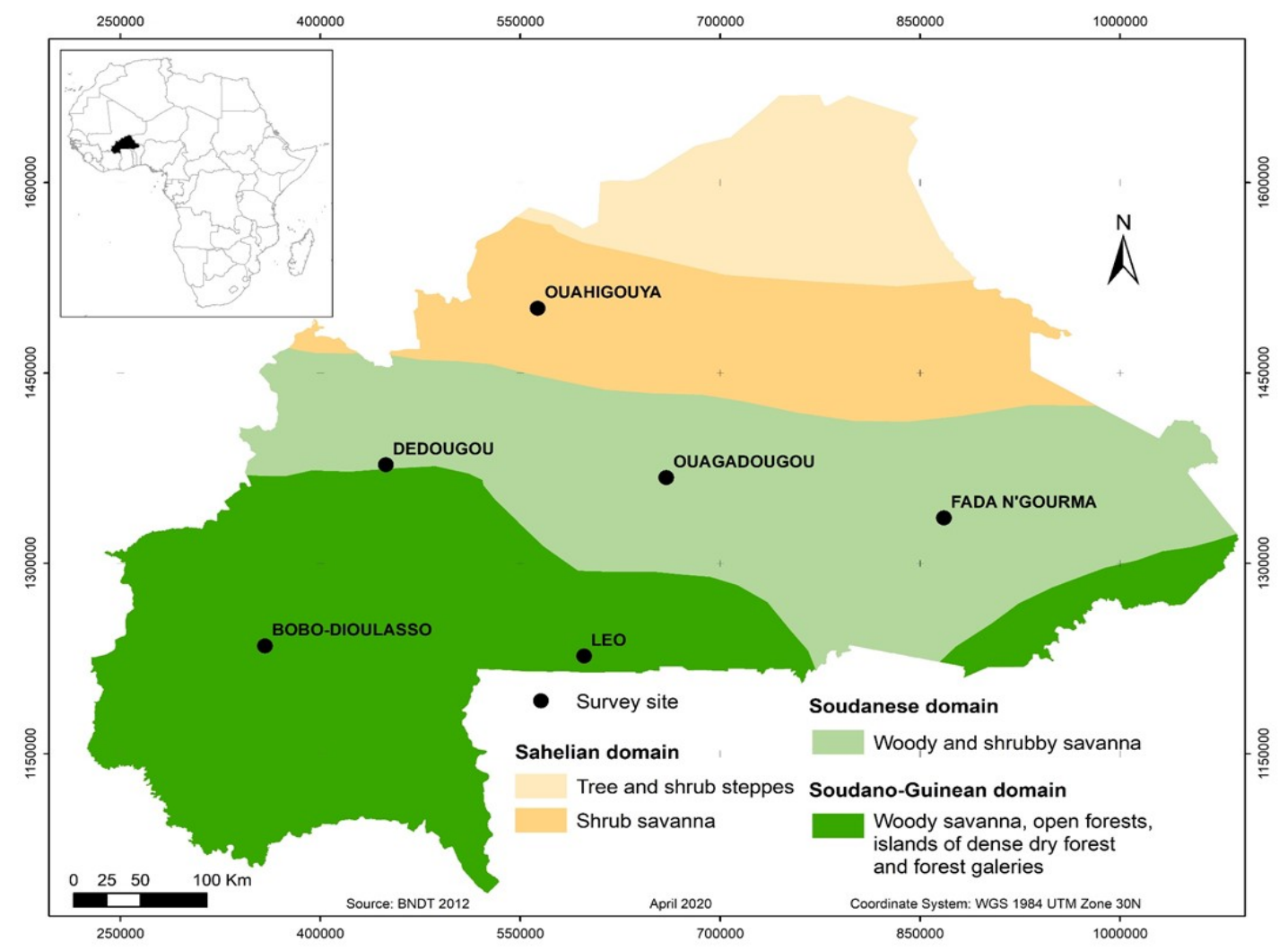

Figure 1. Ethnobotanical surveys sites

\section{Data collection}

This survey targeted herbal traders selling medical plants in the markets. Herbal traders in the surveyed markets belonged mainly to the following ethnic groups: Mossi, Dioula, Gourmantché, Gourounsi, and Fulfudé (FAO 1996). Interviews were conducted in the local language using guided semi-structured questionnaires according to the procedure described by Martin (1995) with slight modification (see in appendix 1 an example questionnaire). The survey was conducted from December 2015 to January 2016. For each market the representative of the sellers of medicinal plants was consulted, who suggested the traders with the most experience in the field of medicinal plant trade or application. Markets were visited once and 1- 2 medicinal plant vendors or traditional healers, who agreed to participate in the survey were interviewed per market, summing up to a total of 30 interviews at 18 local markets. The traders chosen provided information on local names of the plants, plant parts used, quantities offered, prices, plant provenances, methods of preparation, and diseases/symptoms targeted. Botanical samples were acquired from all participants of the survey.

\section{Species identification}

Plant identification was partly carried out in the markets based on field manuals for plant identification encompassing trees, shrubs and lianas from West African dry zones (Arbonnier 2000). In addition, voucher specimens of all plant species were identified at the herbarium "Herbier National du Burkina Faso" (HNBU).

Estimation of plant quantities offered and prices The quantities of plant samples sold in the markets were determined using common estimates based on the hand-circumference of bundles (Figure 2) (Martin 1995) and prices were recorded according to this unit.

\section{Species use value}

The use value of a certain plant species for treating a given category of disease was assessed by asking nine key informants. These were selected on the following criteria i) wide range of plants offered, ii) long experience in medicinal plant trade or application and iii) consent to supply the information. They were asked to assign a value between 1 and 10 (10 for the most frequently used plant) to the ten most available medicinal plant species (Martin 1995). Values assigned to each species were summed up for all informants to get an overall rank value. The species were then ranked in descending order. 


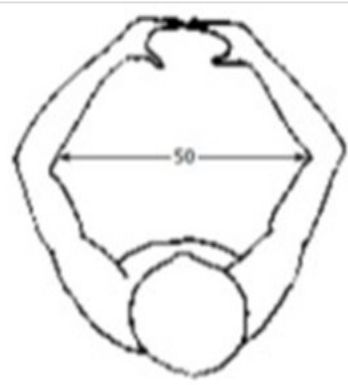

1

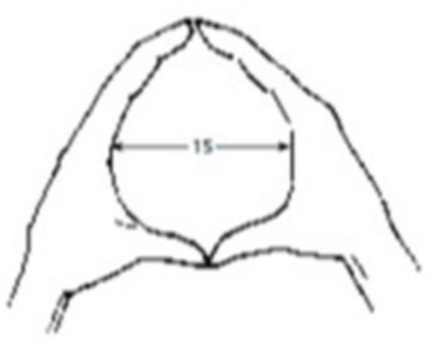

2

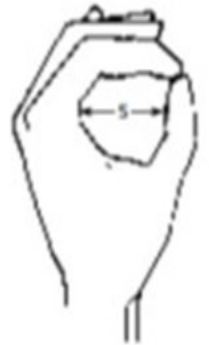

3

Figure 2. Commonly used local estimates of circumference for different-sized bundles of plant products (Martin 1995).

$1=50 \mathrm{~cm}$ of 'arm-circumference' diameters; $2=15 \mathrm{~cm}$ of 'two hands-circumference' diameters; $3=5 \mathrm{~cm}$ of 'one hand-circumference' diameters.

\section{Frequency of citation}

The frequency of the most cited species was calculated according to Dossou et al. (2012) in order to identify the species most valued by the traders.

$$
\mathrm{Fc}=(\mathrm{Nc} / \mathrm{Ns}) \times 100[\%]
$$

Where, Fc = frequency of citation in percentage; Nc $=$ number of traders citing the species and $\mathrm{Ns}=$ total number of traders surveyed.

By convention, a low frequency $(\leq 30 \%)$ indicates that the species is little known and little used. A frequency range between 30 and $60 \%$ denotes that the species is moderately known and moderately used, while a range between 60 and $100 \%$ reveals that the species is very well known and widely used.

\section{Informant consensus factor (FIC)}

The informant consensus factor was calculated according to Uddin and Hassan (2014).

$$
\mathrm{FIC}=\left(\mathrm{N}_{\mathrm{ur}}-\mathrm{N}\right) /\left(\mathrm{N}_{\mathrm{ur}}-1\right)
$$

Where $\mathrm{N}_{\mathrm{ur}}=$ number of use reports for a specific ailment category from vendors in the markets and $\mathrm{N}$ $=$ number of species listed in each category from all vendors.

FIC values can range from 0 (lowest level) to 1 (highest level). FIC values are low when plants are randomly selected or if there is no consensus among informants on their use for the treatment of a certain disease category or if there is no exchange among informants. Values are close to 1 if a large proportion of sellers prefer certain species for a specific disease category or if information is exchanged between informants. In short, medicinal plants with high FIC are believed to be effective in treating a certain disease category.

The main purpose of FIC is to select disease categories for which there is consensus on the use of plants among the informants and to identify species with particular importance in a culture.

\section{Fidelity level (FL)}

The fidelity level (FL) is defined as the ratio between the number of informants who independently suggested the use of a species for the same particular disease and the total number of informants who mentioned the plant for any use (Andrade-Cetto and Heinrich 2011).

$$
F L=\left(I_{p} / I_{u}\right) \times 100[\%]
$$

Where, $I_{p}=$ number of informants using the medicinal plants for a specific disease and $l_{u}=$ number of informants reporting the use of the species for any ailment.

\section{Conservation status of medicinal plants in the markets}

The conservation status of all species recorded was assessed on the basis of the IUCN red list database (IUCN 2018).

\section{Data analysis}

Data collected were tabulated and analyzed by using Microsoft Excel. For calculation of the informant consensus factor the ailments/symptoms treated by the medicinal plants were grouped into 13 different categories according to the Economic Botany Data Collection Standard with slight modifications (IwU 1993). 


\section{Results}

Information on vendors and plant taxa sold

Of the 30 vendors interviewed in 18 markets, women predominated, accounting for $87 \%$ of the vendors. The age of the informants ranged between 45 and 64 years. Twenty vendors were affiliated to an association involved in the field of traditional medicine and ten vendors had no affiliation. Half of the informants had received formal training from private schools and non-governmental organizations involved in the area of medicinal plants.

In total, 104 species of medicinal plants distributed in 39 families and 81 genera could be identified in the survey (Table 1). The dominant plant families were Fabaceae (16 species), followed by Rubiaceae ( 7 species), and Combretaceae (5 species). Twentyone species were present in at least $50 \%$ of the stalls, 38 species were sold only by $3 \%$ of the vendors (Table 1). The highest numbers of different medicinal plant species were encountered in the two largest cities of Burkina Faso, in Ougadougou ( $n=48)$ and Bobo-Dioulasso $(n=40)$. The most unique species collection in relation to the total number of species offered was found in the cities of Fada N'Gourma and Léo: approximately $50 \%$ of the species offered in these two cities were only found there.

\section{Plant parts, provenance and growth forms of plants used for herbal preparations}

The most commonly used plant parts were leaves with a proportion of $47.0 \%$ of all nominations (Table 2 ), followed by stem bark (34.0\%) and roots (17.2\%). Inflorescences, gums and seeds were rarely used. In many cases, more than one part of the same plant species was used, for instance often leaves, stem bark and roots of the same species were employed to prepare remedies. Most of the medicinal plants sold on the stalls came from the savanna $(86.3 \%$ of the samples, Table 3). The species collected in the forests included only trees with a proportion of $2.6 \%$. Ligneous material from fields or gardens, respectively, and herbs from gardens comprised only a very small proportion of the material found in the markets.

Only rarely medicinal plant species sold in the markets came from cultivated sources, such as hedgerows (Bauhinia rufescens Lam., Vachellia nilotica (L.) P.J.H. Hurter \& Mabb.), or home gardens (B. rufescens, Cymbopogon citratus (DC.) Stapf, Moringa oleïfera Lam.).

\section{Medicinal plants recipe preparation and administration}

We recorded six types of methods for recipe preparation, namely decoction, trituration, infusion, cataplasm, calcination and crushed mixture with water. Decoction was the most common method of preparation $(87.5 \%)$, followed by trituration $(14.4 \%)$ and infusion (12.5\%) (Figure 3). The medicinal plant preparations were administered through different routes including drink, bath, gargle, purgative, application, massage, inhalation, toothbrush, instillation and enema. The main mode of administration was the drink $(65.4 \%)$ followed by the bath (35.6\%) (Figure 4).

\section{Range of ailments treated by medicinal plants}

According to the information supplied by the vendors a total of 31 conditions were treated with medicinal plants. In order to calculate various ethnobotanical indices these conditions were classified into 13 disease categories. First the number of species used for treatment of a certain disease categories was analyzed (Table 4). The largest number of plant species was used for treatment of gastrointestinal diseases $(48.1 \%)$ followed by malaria (19.2\%), general pathology symptoms (18.3) dermatosis and respiratory diseases (each $17.3 \%$ ). The range of disease categories treated by a particular species varied from 1 to 4 . The species for which the most diverse healing potential was assigned by the traders were Phyllanthus amarus Schum. \& Thonn. (gastrointestinal diseases, hemorrhoids, liver disorders, arthritis) and Moringa oleïfera Lam. (gastrointestinal diseases, malaria, general pathology symptoms, cardiovascular diseases).

\section{Factor of informant consensus (FIC)}

For this factor calculation, the 13 disease categories as defined in Table 4 were used. Exceptional high consensus about plant usage among informants was found for childhood illnesses $(F I C=0.9)$ and cardiovascular diseases $(F I C=0.8)$ followed by respiratory diseases, general pathology symptoms, arthritis and other infections (each $\mathrm{FIC}=0.7$ ) (Table $5)$. Very low FIC values $(\leq 0.4)$, as observed for urogenital and internal organ diseases, indicate that informants mostly disagreed on which plants to use for these diseases. The most important plants used for the category of childhood illnesses were Lepidagathis anobrya (Pers.) Decne. Walteria indica L., and Ocimum basilicum L. (Table 1). For the category of cardiovascular diseases, Mitragyna inermis (Willd.) Kuntze was the species most cited. 
Table 1. List of medicinal plants reported in the markets of six cities of Burkina Faso

\begin{tabular}{|c|c|c|c|c|c|c|c|c|c|c|c|}
\hline \multirow{2}{*}{$\begin{array}{l}\text { Family, Scientific name } \\
\text { Voucher specimen number }\end{array}$} & \multirow[t]{2}{*}{ Local name } & \multirow[t]{2}{*}{ City } & \multicolumn{2}{|c|}{ Provenance } & \multirow{2}{*}{$\begin{array}{l}\text { Part } \\
\text { used }\end{array}$} & \multirow[t]{2}{*}{ Preparation } & \multirow{2}{*}{$\begin{array}{l}\text { Ailment/ } \\
\text { Symptoms } \\
\text { Treated }\end{array}$} & \multirow[t]{2}{*}{ Administration } & \multirow{2}{*}{$\begin{array}{l}\text { Frequency of } \\
\text { citation (\%) }\end{array}$} & \multirow{2}{*}{$\begin{array}{l}\text { Estimated } \\
\text { quantity by hand- } \\
\text { circumference } \\
\text { (average quantity } \\
\text { offered by the } \\
\text { vendors) }\end{array}$} & \multirow{2}{*}{$\begin{array}{l}\text { Price by hand- } \\
\text { circumference } \\
\text { [FCFA] }\end{array}$} \\
\hline & & & Savanna & Forest & & & & & & & \\
\hline \multicolumn{12}{|l|}{ Acanthaceae } \\
\hline $\begin{array}{l}\text { Hygrophila auriculata (Schum.) } \\
\text { Heine } \\
2243\end{array}$ & Kocenkoéré (M) & 2 & $\mathrm{H}$ & $\mathrm{NI}$ & $\mathrm{Sb}$ & Trituration & $\begin{array}{l}\text { Respiratory } \\
\text { diseases }\end{array}$ & Drink & 10 & $\begin{array}{l}\text { Two hands- } \\
\text { circumference of } \\
\text { stem }\end{array}$ & 100 \\
\hline $\begin{array}{l}\text { Lepidagathis anobrya Nees } \\
540\end{array}$ & $\begin{array}{l}\text { Gnuy-nakida } \\
\text { (M), Gonwou } \\
\text { nouman (D) }\end{array}$ & $1,2,3$ & $\mathrm{H}$ & $\mathrm{NI}$ & L & Decoction & $\begin{array}{l}\text { Growth } \\
\text { retardation, } \\
\text { stomachache }\end{array}$ & Drink & 50 & $\begin{array}{l}\text { One hand- } \\
\text { circumference of } \\
\text { plant }\end{array}$ & 50 \\
\hline \multicolumn{12}{|l|}{ Amaranthaceae } \\
\hline $\begin{array}{l}\text { Alternanthera nodiflora R. Br. } \\
2055\end{array}$ & Sibgponré (M) & 1 & $\mathrm{H}$ & $\mathrm{NI}$ & L & Cataplasm & $\begin{array}{l}\text { Growth } \\
\text { retardation }\end{array}$ & Purgative & 17 & $\begin{array}{l}\text { One hand- } \\
\text { circumference of } \\
\text { plant }\end{array}$ & 50 \\
\hline $\begin{array}{l}\text { Pupalia lappacea (L.) Juss. } \\
5599\end{array}$ & Nwestabdo (M) & 1 & $\mathrm{H}$ & $\mathrm{NI}$ & L & Calcination & $\begin{array}{l}\text { Wound healing } \\
\text { stomachache }\end{array}$ & Application & 3 & $\begin{array}{l}\text { One hand- } \\
\text { circumference of } \\
\text { plant }\end{array}$ & 50 \\
\hline \multicolumn{12}{|l|}{ Anacardiaceae } \\
\hline $\begin{array}{l}\text { Anacardium occidentale L. } \\
2010\end{array}$ & Finzan(D) & 2 & $\mathrm{Li}$ & $\mathrm{NI}$ & $\mathrm{Sb}$ & Infusion & Hypertension & Drink & 7 & $\begin{array}{l}\text { One hand- } \\
\text { circumference } \\
\text { stem bark }\end{array}$ & 50 \\
\hline $\begin{array}{l}\text { Lannea acida A. Rich. } \\
2596\end{array}$ & $\begin{array}{l}\text { Sabtulga (M), } \\
\text { Eberg }(G)\end{array}$ & 3,4 & $\mathrm{Li}$ & $\mathrm{NI}$ & $\begin{array}{l}\mathrm{Sb} \\
\mathrm{R}\end{array}$ & Decoction & $\begin{array}{l}\text { Stomachache, } \\
\text { ulcer }\end{array}$ & Drink & 3 & $\begin{array}{l}\text { One hand- } \\
\text { circumference } \\
\text { stem bark }\end{array}$ & 50 \\
\hline $\begin{array}{l}\text { Lannea velutina A. Rich. } \\
8203\end{array}$ & $\begin{array}{l}\text { Wâamsâbga } \\
\text { (M) }\end{array}$ & 6 & $\mathrm{Li}$ & $\mathrm{NI}$ & $\mathrm{L}, \mathrm{Sb}$ & Decoction & Tonic & Bath & 3 & $\begin{array}{l}\text { Two hands- } \\
\text { circumference of } \\
\text { leafy stem }\end{array}$ & 50 \\
\hline $\begin{array}{l}\text { Sclerocarya birrea (A. Rich.) } \\
\text { Hochst. } \\
\text { N360 }\end{array}$ & Noabega (M) & 2,4 & $\mathrm{Li}$ & $\mathrm{NI}$ & $\mathrm{Sb}$ & $\begin{array}{l}\text { Decoction, } \\
\text { infusion }\end{array}$ & Dysentery, Ulcer & Drink & 50 & $\begin{array}{l}\text { One hand- } \\
\text { circumference of } \\
\text { stem bark }\end{array}$ & 50 \\
\hline \multicolumn{12}{|l|}{ Annonaceae } \\
\hline $\begin{array}{l}\text { Annona senegalensis Pers. } \\
2083\end{array}$ & $\begin{array}{l}\text { Sunsun (D), } \\
\text { Barkudga (M) }\end{array}$ & $1,2,3,5$ & $\mathrm{Li}$ & $\mathrm{NI}$ & L, Sb, R & $\begin{array}{l}\text { Decoction, } \\
\text { calcination }\end{array}$ & $\begin{array}{l}\text { Wound healing, } \\
\text { stomachache, } \\
\text { somniferous } \\
\text { malaria }\end{array}$ & $\begin{array}{l}\text { Drink, bath, } \\
\text { application, enema }\end{array}$ & 67 & $\begin{array}{l}\text { One hand- } \\
\text { circumference of } \\
\text { leafy stem }\end{array}$ & 50 \\
\hline
\end{tabular}




\begin{tabular}{|c|c|c|c|c|c|c|c|c|c|c|c|}
\hline $\begin{array}{l}\text { Baissea multiflora A.DC. } \\
8403\end{array}$ & $\begin{array}{l}\text { Nonguem taaba, } \\
\text { Nongsego (M) }\end{array}$ & 2 & $\mathrm{Li} / \mathrm{La}$ & $\mathrm{NI}$ & $L, S b, R$ & Decoction & Tonic, diarrhea & Drink & 10 & $\begin{array}{l}\text { Two hands- } \\
\text { circumference of } \\
\text { leafy stem }\end{array}$ & 50 \\
\hline \multicolumn{12}{|l|}{ Apocynaceae } \\
\hline $\begin{array}{l}\text { Saba senegalensis (A. DC.) } \\
\text { Pichon } \\
2741\end{array}$ & $\begin{array}{l}\text { Wêdga (M), } \\
\text { Zaban(D) }\end{array}$ & $1,3,5$ & $\mathrm{Li}$ & $\mathrm{NI}$ & L & Decoction & $\begin{array}{l}\text { Wound healing, } \\
\text { stomachache }\end{array}$ & Drink, enema & 50 & $\begin{array}{l}\text { Two hands- } \\
\text { circumference of } \\
\text { leafy stem }\end{array}$ & 50 \\
\hline $\begin{array}{l}\text { Cynanchum viminale (L.) L. } \\
2016\end{array}$ & Wobsaré (M) & 3,5 & Li/La & $\mathrm{NI}$ & $\mathrm{Sb}, \mathrm{Sa}$ & Trituration & Eye infection & $\begin{array}{l}\text { Instillation, } \\
\text { application }\end{array}$ & 50 & $\begin{array}{l}\text { Two hands- } \\
\text { circumference of } \\
\text { leafy stem }\end{array}$ & 100 \\
\hline $\begin{array}{l}\text { Leptadenia hastata (Pers.) } \\
\text { Decne. } \\
2060\end{array}$ & Lelungo (M) & 3 & Li/la & $\mathrm{NI}$ & L, Sb & Decoction & $\begin{array}{l}\text { Malaria, } \\
\text { jaundice, sexual } \\
\text { impotence }\end{array}$ & Drink & 7 & $\begin{array}{l}\text { One hand- } \\
\text { circumference of } \\
\text { leafy stem }\end{array}$ & 50 \\
\hline \multicolumn{12}{|l|}{ Asteraceae } \\
\hline $\begin{array}{l}\text { Chrysanthellum americanum (L.) } \\
\text { Vatke } \\
5464\end{array}$ & Waltuko (M) & 1 & $\mathrm{H}$ & $\mathrm{NI}$ & L, Sb, R & $\begin{array}{l}\text { Decoction, } \\
\text { infusion }\end{array}$ & Liver diseases & Drink & 7 & $\begin{array}{l}\text { One hand- } \\
\text { circumference of } \\
\text { the plant }\end{array}$ & $50-200$ \\
\hline \multicolumn{12}{|l|}{ Bombacaceae } \\
\hline $\begin{array}{l}\text { Adansonia digitata L. } \\
2038\end{array}$ & Toêga (M) & 6 & $\mathrm{Li}$ & $\mathrm{NI}$ & L & Decoction & Dysentery & Drink & 3 & $\begin{array}{l}\text { Two hands- } \\
\text { circumference of } \\
\text { leaf }\end{array}$ & 50 \\
\hline \multicolumn{12}{|l|}{ Boraginaceae } \\
\hline $\begin{array}{l}\text { Heliotropium } \\
\text { indicum L. } \\
3070\end{array}$ & Nonsikou (D) & 2 & $\mathrm{H}$ & $\mathrm{NI}$ & L, Sb & Decoction & $\begin{array}{l}\text { Diarrhea, } \\
\text { hypertension }\end{array}$ & Drink & 3 & $\begin{array}{l}\text { One hand- } \\
\text { circumference of } \\
\text { plant }\end{array}$ & 50 \\
\hline Burseraceae & & & & $\mathrm{NI}$ & & & & & & & \\
\hline $\begin{array}{l}\text { Boswellia dalzielii Hutch. } \\
8103\end{array}$ & $\begin{array}{l}\text { Gondreyongo } \\
\text { (M) }\end{array}$ & 1 & $\mathrm{Li}$ & $\mathrm{NI}$ & $L$ & Decoction & $\begin{array}{l}\text { Wound healing, } \\
\text { stomachache }\end{array}$ & Enema & 10 & $\begin{array}{l}\text { One hand- } \\
\text { circumference of } \\
\text { leafy stem }\end{array}$ & 50 \\
\hline \multicolumn{12}{|l|}{ Celastraceae } \\
\hline $\begin{array}{l}\text { Maytenus senegalensis (Lam.) } \\
\text { Exell } \\
2247\end{array}$ & $\begin{array}{l}\text { Tovougri (M), } \\
\text { Barkawouli (Gsi) }\end{array}$ & $1,3,6$ & $\mathrm{Li}$ & $\mathrm{NI}$ & $L, R$ & Decoction & $\begin{array}{l}\text { Malaria, } \\
\text { diarrhea, dental } \\
\text { pain, headache }\end{array}$ & $\begin{array}{l}\text { Bath, } \\
\text { gargle }\end{array}$ & 50 & $\begin{array}{l}\text { One hand- } \\
\text { circumference of } \\
\text { leafy stem }\end{array}$ & 50 \\
\hline $\begin{array}{l}\text { Hippocratea africana (Willd.) } \\
\text { Loes. } \\
2446\end{array}$ & Zibri (M) & 3 & $\mathrm{Li}$ & $\mathrm{NI}$ & $\mathrm{Sb}$ & Decoction & $\begin{array}{l}\text { Sickle-cell } \\
\text { disease }\end{array}$ & Bath & 3 & $\begin{array}{l}\text { Two hands- } \\
\text { circumference of } \\
\text { leaf }\end{array}$ & 50 \\
\hline
\end{tabular}




\begin{tabular}{|c|c|c|c|c|c|c|c|c|c|c|c|}
\hline \multicolumn{12}{|l|}{ Cochlospermaceae } \\
\hline $\begin{array}{l}\text { Cochlospermum planchonii } \\
\text { Hook.f. } \\
8437\end{array}$ & Sons-raaga (M) & 5 & $\mathrm{Li}$ & $\mathrm{Li}$ & $\mathrm{R}$ & Decoction & Malaria & Drink, bath & 17 & $\begin{array}{l}\text { One hand- } \\
\text { circumference of } \\
\text { the root }\end{array}$ & 50 \\
\hline $\begin{array}{l}\text { Cochlospermum tinctorium } \\
\text { Perrier ex A. Rich. } \\
3430\end{array}$ & N'dribala (D) & 2 & $\mathrm{Li}$ & $\mathrm{NI}$ & L,R & Decoction & Malaria, ulcer & Drink, bath & 17 & $\begin{array}{l}\text { Two hands- } \\
\text { circumference of } \\
\text { leafy stem }\end{array}$ & 100 \\
\hline \multicolumn{12}{|l|}{ Combretaceae } \\
\hline $\begin{array}{l}\text { Anogeissus leiocarpa (DC.) } \\
\text { Guill. \& Perr. } \\
5799\end{array}$ & Siiga (M) & 2 & $\mathrm{Li}$ & $\mathrm{NI}$ & $\mathrm{Sb}$ & Decoction & $\begin{array}{l}\text { Respiratory } \\
\text { diseases }\end{array}$ & Drink & 33 & $\begin{array}{l}\text { Two hands- } \\
\text { circumference of } \\
\text { stem bark }\end{array}$ & 50 \\
\hline $\begin{array}{l}\text { Combretum aculeatum Vent. } \\
1904\end{array}$ & Guitga (M) & $1,4,6$ & $\mathrm{Li}$ & $\mathrm{NI}$ & L & Decoction & Dental pain & Gargle & 50 & $\begin{array}{l}\text { Two hands- } \\
\text { circumference of } \\
\text { leaf }\end{array}$ & 50 \\
\hline $\begin{array}{l}\text { Combretum adenogonium } \\
\text { Steud. ex A. Rich. } \\
2244\end{array}$ & $\begin{array}{l}\text { Dayigoubwala } \\
\text { (Gsi),Kwigenga } \\
\text { (M) }\end{array}$ & 5,6 & $\mathrm{Li}$ & $\mathrm{NI}$ & $\mathrm{L}, \mathrm{Sb}$ & Decoction & Dysentery & Drink & 3 & $\begin{array}{l}\text { Two hands- } \\
\text { circumference of } \\
\text { leaf }\end{array}$ & 50 \\
\hline $\begin{array}{l}\text { Combretum glutinosum Perr. ex } \\
\text { DC. } \\
2263\end{array}$ & $\begin{array}{l}\text { Kuenga (M), } \\
\text { Lifapelu(G) }\end{array}$ & 4 & $\mathrm{Li}$ & $\mathrm{NI}$ & $\mathrm{L}$ & Decoction & $\begin{array}{l}\text { Stomachache, } \\
\text { liver trouble }\end{array}$ & Drink & 3 & $\begin{array}{l}\text { Two hands- } \\
\text { circumference of } \\
\text { leaf }\end{array}$ & 50 \\
\hline $\begin{array}{l}\text { Combretum micranthum G. Don } \\
346\end{array}$ & Raandga (M) & $2,3,4$ & $\mathrm{Li}$ & $\mathrm{NI}$ & L & Decoction & Malaria & Drink & 50 & $\begin{array}{l}\text { Two hands- } \\
\text { circumference of } \\
\text { leaf }\end{array}$ & 50 \\
\hline $\begin{array}{l}\text { Combretum paniculatum Vent. } \\
2250\end{array}$ & $\begin{array}{l}\text { Kudgunlungu } \\
\text { (M) }\end{array}$ & 3 & $\mathrm{Li}$ & $\mathrm{NI}$ & L & $\begin{array}{l}\text { Decoction, } \\
\text { maceration }\end{array}$ & Hemorrhoids & Drink & 7 & $\begin{array}{l}\text { Two hands- } \\
\text { circumference of } \\
\text { leaf }\end{array}$ & 50 \\
\hline $\begin{array}{l}\text { Combretum sericeum G. Don } \\
340\end{array}$ & Vopopwao (Gsi) & 6 & $\mathrm{Li}$ & $\mathrm{NI}$ & $\mathrm{L}, \mathrm{Sb}$ & Decoction & General fatigue & Bath & 3 & $\begin{array}{l}\text { Two hands- } \\
\text { circumference of } \\
\text { leaf }\end{array}$ & 50 \\
\hline $\begin{array}{l}\text { Guiera senegalensis J.F. Gmel. } \\
1921\end{array}$ & Wilim-wiiga (M) & $1,2,3$ & $\mathrm{Li}$ & $\mathrm{NI}$ & $\mathrm{L}, \mathrm{Sb}$ & Decoction & $\begin{array}{l}\text { Malaria, } \\
\text { diarrhea, cold, } \\
\text { sinusitis }\end{array}$ & $\begin{array}{l}\text { Drink, } \\
\text { bath }\end{array}$ & 50 & $\begin{array}{l}\text { Two hands- } \\
\text { circumference of } \\
\text { leaf }\end{array}$ & 50 \\
\hline $\begin{array}{l}\text { Pteleopsis suberosa Engl. \& } \\
\text { Diels } \\
1876\end{array}$ & $\begin{array}{l}\text { Guirga (M), } \\
\text { Gnonlifou (D) }\end{array}$ & $1,2,4,5$ & $\mathrm{Li}$ & $\mathrm{NI}$ & $\mathrm{L}, \mathrm{Sb}$ & $\begin{array}{l}\text { Decoction, } \\
\text { maceration }\end{array}$ & $\begin{array}{l}\text { Dysentery, } \\
\text { stomachache, } \\
\text { cough, } \\
\text { hemorrhoids }\end{array}$ & Drink & 67 & $\begin{array}{l}\text { Two hands- } \\
\text { circumference of } \\
\text { leaf }\end{array}$ & 50 \\
\hline $\begin{array}{l}\text { Terminalia avicennioïdes Guill. \& } \\
\text { Perr. } \\
2091\end{array}$ & Kondré (M) & 2 & $\mathrm{Li}$ & $\mathrm{NI}$ & $\mathrm{L}, \mathrm{Sb}, \mathrm{R}$ & Decoction & $\begin{array}{l}\text { Liver trouble, } \\
\text { heartache, } \\
\text { diarrhea }\end{array}$ & Drink & 50 & $\begin{array}{l}\text { Two hands- } \\
\text { circumference of } \\
\text { leaf }\end{array}$ & 50 \\
\hline
\end{tabular}




\begin{tabular}{|c|c|c|c|c|c|c|c|c|c|c|c|}
\hline $\begin{array}{l}\text { Terminalia macroptera Guill. \& } \\
\text { Perr. } \\
2007\end{array}$ & Koond-poko (M) & 1 & $\mathrm{Li}$ & $\mathrm{NI}$ & $\mathrm{R}$ & Decoction & Hemorrhoids & Enema & 3 & $\begin{array}{l}\text { One hands- } \\
\text { circumference of } \\
\text { root }\end{array}$ & 50 \\
\hline \multicolumn{12}{|l|}{ Ebenaceae } \\
\hline $\begin{array}{l}\text { Diospyros mespiliformis Hochst. } \\
\text { ex A. DC. } \\
4637\end{array}$ & Gaaka (M) & 1,4 & Li & $\mathrm{Li}$ & $\mathrm{L}, \mathrm{Sb}, \mathrm{R}$ & Decoction & $\begin{array}{l}\text { Wound healing, } \\
\text { sexual } \\
\text { impotence, } \\
\text { parasite } \\
\text { infections }\end{array}$ & $\begin{array}{l}\text { Drink, } \\
\text { bath }\end{array}$ & 17 & $\begin{array}{l}\text { Two hands- } \\
\text { circumference of } \\
\text { leaf }\end{array}$ & 50 \\
\hline \multicolumn{12}{|l|}{ Euphorbiaceae } \\
\hline $\begin{array}{l}\text { Euphorbia hirta L. } \\
468\end{array}$ & Wal-biisum (M) & 1 & $\mathrm{H}$ & $\mathrm{NI}$ & $\mathrm{L}, \mathrm{Sb}, \mathrm{R}$ & $\begin{array}{l}\text { Maceration, } \\
\text { decoction }\end{array}$ & $\begin{array}{l}\text { Dysentery, } \\
\text { respiratory } \\
\text { diseases }\end{array}$ & Drink & 7 & $\begin{array}{l}\text { One hand- } \\
\text { circumference of } \\
\text { plant }\end{array}$ & 50 \\
\hline Fabaceae & & & & $\mathrm{NI}$ & & & & & & & \\
\hline $\begin{array}{l}\text { Afzelia africana Smith ex Pers. } \\
2414\end{array}$ & $\begin{array}{l}\text { Kankalga (M), } \\
\text { Tiégué (Gsi) }\end{array}$ & 6 & $\mathrm{NI}$ & Li & $\begin{array}{l}\mathrm{Sb} \\
\mathrm{R}\end{array}$ & Decoction & Stomachache & Drink & 3 & $\begin{array}{l}\text { One hand- } \\
\text { circumference of } \\
\text { stem bark }\end{array}$ & 50 \\
\hline $\begin{array}{l}\text { Alysicarpus glumaceus (Vahl) } \\
\text { DC. } \\
2402\end{array}$ & Rabgo (M) & 5 & $\mathrm{Hs}$ & $\mathrm{NI}$ & $\mathrm{L}$ & Decoction & Diarrhea & Drink & 3 & $\begin{array}{l}\text { One hand- } \\
\text { circumference of } \\
\text { the plant }\end{array}$ & 50 \\
\hline $\begin{array}{l}\text { Bauhinia rufescens } \\
\text { Lam. } \\
6045\end{array}$ & Tipohèga (M) & 1,2 & $\mathrm{Li} / \mathrm{He}$ & $\mathrm{NI}$ & $\begin{array}{l}\mathrm{L}, \mathrm{Sb}, \mathrm{R}, \\
\mathrm{Fr}\end{array}$ & Trituration & $\begin{array}{l}\text { Cold, sinusitis, } \\
\text { tonic }\end{array}$ & Fumigation, bath & 50 & $\begin{array}{l}\text { One hand- } \\
\text { circumference of } \\
\text { leaf }\end{array}$ & 50 \\
\hline $\begin{array}{l}\text { Burkea africana Hook. } \\
1994\end{array}$ & Kasi-sané (M) & 6 & $\mathrm{Li}$ & Li & $\mathrm{Sb}$ & $\begin{array}{l}\text { Decoction, } \\
\text { infusion }\end{array}$ & $\begin{array}{l}\text { Cough, heart } \\
\text { diseases }\end{array}$ & Drink & 3 & $\begin{array}{l}\text { One hand- } \\
\text { circumference of } \\
\text { stem bark }\end{array}$ & 50 \\
\hline $\begin{array}{l}\text { Cassia alata L. } \\
220\end{array}$ & Colbata (D) & 2 & $\mathrm{Li}$ & $\mathrm{NI}$ & L & $\begin{array}{l}\text { Decoction, } \\
\text { trituration }\end{array}$ & $\begin{array}{l}\text { Skin disorders, } \\
\text { liver diseases }\end{array}$ & Drink, application & 3 & $\begin{array}{l}\text { One hand- } \\
\text { circumference of } \\
\text { leafy stem }\end{array}$ & 50 \\
\hline $\begin{array}{l}\text { Cassia italica (Mill.) Lam. ex } \\
\text { F.W. Andrews } \\
232\end{array}$ & $\begin{array}{l}\text { Panedmantiiga } \\
\text { (M), Bali bali (D) }\end{array}$ & 1 & $\mathrm{Li}$ & $\mathrm{NI}$ & $\mathrm{L}$ & Decoction & $\begin{array}{l}\text { Stomachache, } \\
\text { skin disorders }\end{array}$ & Drink, bath & 17 & $\begin{array}{l}\text { One hand- } \\
\text { circumference of } \\
\text { leafy stem }\end{array}$ & 50 \\
\hline $\begin{array}{l}\text { Cassia nigricans Vahl } \\
2223\end{array}$ & Zander-kuka (M) & 1 & $\mathrm{Li}$ & $\mathrm{NI}$ & $\mathrm{L}$ & $\begin{array}{l}\text { Decoction, } \\
\text { trituration }\end{array}$ & $\begin{array}{l}\text { Wound healing, } \\
\text { stomachache }\end{array}$ & Enema, application & 10 & $\begin{array}{l}\text { One hand- } \\
\text { circumference of } \\
\text { leafy stem }\end{array}$ & 50 \\
\hline $\begin{array}{l}\text { Cassia occidentalis L. } \\
5375\end{array}$ & Balambala (D) & 5 & $\mathrm{Li}$ & $\mathrm{NI}$ & L, Sb & Decoction & $\begin{array}{l}\text { Tonic, } \\
\text { stomachache }\end{array}$ & Bath & 13 & $\begin{array}{l}\text { One hand- } \\
\text { circumference of } \\
\text { leafy stem }\end{array}$ & 50 \\
\hline
\end{tabular}




\begin{tabular}{|c|c|c|c|c|c|c|c|c|c|c|c|}
\hline $\begin{array}{l}\text { Cassia sieberiana DC. } \\
5895\end{array}$ & $\begin{array}{l}\text { Samdiani (G), } \\
\text { Kumbr-saka (M) }\end{array}$ & $1,3,4$ & $\mathrm{Li}$ & $\mathrm{NI}$ & $\begin{array}{l}\mathrm{L}, \mathrm{Sb}, \\
\text { Root }\end{array}$ & $\begin{array}{l}\text { Decoction, } \\
\text { trituration }\end{array}$ & $\begin{array}{l}\text { Stomachache, } \\
\text { malaria, kidney } \\
\text { diseases, sexual } \\
\text { impotence }\end{array}$ & Drink & 50 & $\begin{array}{l}\text { One hand- } \\
\text { circumference of } \\
\text { leafy stem }\end{array}$ & $50-100$ \\
\hline $\begin{array}{l}\text { Cassia singueana Del. } \\
3113\end{array}$ & Djélponsré (M) & 1 & $\mathrm{Li}$ & $\mathrm{NI}$ & $L, R$ & Decoction & Stomachache & Drink & 10 & $\begin{array}{l}\text { One hand- } \\
\text { circumference of } \\
\text { leafy stem }\end{array}$ & 50 \\
\hline $\begin{array}{l}\text { Detarium microcarpum Guill. \& } \\
\text { Perr. } \\
2948\end{array}$ & Kadga (M) & 4 & $\mathrm{Li}$ & $\mathrm{Li}$ & $\mathrm{L}, \mathrm{Sb}$ & Decoction & $\begin{array}{l}\text { Headache, } \\
\text { stomachache }\end{array}$ & Drink & 3 & $\begin{array}{l}\text { One hand- } \\
\text { circumference of } \\
\text { leafy stem }\end{array}$ & 50 \\
\hline $\begin{array}{l}\text { Dichrostachys cinerea (L.) Wight } \\
\text { \& Arn. } \\
2678\end{array}$ & Susutga (M) & 6 & $\mathrm{Li}$ & $\mathrm{NI}$ & $\mathrm{R}$ & Decoction & $\begin{array}{l}\text { Respiratory } \\
\text { diseases }\end{array}$ & Drink & 3 & $\begin{array}{l}\text { One hand- } \\
\text { circumference of } \\
\text { stem bark }\end{array}$ & 50 \\
\hline $\begin{array}{l}\text { Entada africana Guill. \& Perr. } \\
2993\end{array}$ & Sennogo (M) & 2 & $\mathrm{NI}$ & $\mathrm{Li}$ & $\mathrm{Sb}$ & $\begin{array}{l}\text { Decoction, } \\
\text { maceration }\end{array}$ & $\begin{array}{l}\text { Cold, sinusitis, } \\
\text { cough }\end{array}$ & Drink & 3 & $\begin{array}{l}\text { Two hands- } \\
\text { circumference of } \\
\text { stem bark }\end{array}$ & 50 \\
\hline $\begin{array}{l}\text { Faidherbia albida (Del.) A. } \\
\text { Chev. } \\
420\end{array}$ & Zaanga (M) & $1,2,3$ & $\mathrm{Li}$ & $\mathrm{NI}$ & $\mathrm{L}$ & Decoction & $\begin{array}{l}\text { Kidney diseases, } \\
\text { malaria }\end{array}$ & Drink & 3 & $\begin{array}{l}\text { One hand- } \\
\text { circumference of } \\
\text { leafy stem }\end{array}$ & 50 \\
\hline $\begin{array}{l}\text { Senegalia macrostachya (Rchb. } \\
\text { ex DC.) Kyal \& Boatwr. } \\
52\end{array}$ & Zamenega (M) & 1 & $\mathrm{Li}$ & $\mathrm{NI}$ & Le & Decoction & Headache & $\begin{array}{l}\text { Drink, } \\
\text { bath }\end{array}$ & 3 & $\begin{array}{l}\text { Two hands- } \\
\text { circumference of } \\
\text { leafy stem }\end{array}$ & 50 \\
\hline $\begin{array}{l}\text { Senegalia senegal (L.) Britton } \\
8609\end{array}$ & Gompèlga (M) & 1 & $\mathrm{Li}$ & $\mathrm{NI}$ & $\mathrm{L}, \mathrm{Sb}$ & Decoction & $\begin{array}{l}\text { Diarrhea, } \\
\text { hemorrhoids }\end{array}$ & Drink & 3 & $\begin{array}{l}\text { Two hands- } \\
\text { circumference of } \\
\text { leafy stem }\end{array}$ & 50 \\
\hline $\begin{array}{l}\text { Vachellia nilotica (L.) P.J.H. } \\
\text { Hurter \& Mabb. } \\
680\end{array}$ & Pegengá (M) & $1,3,6$ & $\mathrm{Li}$ & $\mathrm{NI}$ & $\mathrm{Fr}, \mathrm{Fr}, \mathrm{Sb}$ & Decoction & $\begin{array}{l}\text { Stomachache, } \\
\text { hemorrhoids, } \\
\text { cough }\end{array}$ & Drink, bath, gargle & 50 & $\begin{array}{l}\text { Two hands- } \\
\text { circumference of } \\
\text { leafy stem }\end{array}$ & 50 \\
\hline $\begin{array}{l}\text { Parkia biglobosa (Jacq.) R. Br. } \\
\text { ex G. Don } \\
669\end{array}$ & Rondo (M) & 1,3 & $\mathrm{Li}$ & $\mathrm{NI}$ & $\mathrm{Sb}, \mathrm{Fr}$ & $\begin{array}{l}\text { Decoction, } \\
\text { calcination, } \\
\text { trituration }\end{array}$ & $\begin{array}{l}\text { Wound healing, } \\
\text { hemorrhoids }\end{array}$ & Drink & 27 & $\begin{array}{l}\text { Two hands- } \\
\text { circumference of } \\
\text { stem bark }\end{array}$ & 100 \\
\hline $\begin{array}{l}\text { Piliostigma reticulatum (DC.) } \\
\text { Hochst. } \\
5329\end{array}$ & Bâguendé (M) & $1,2,3,4$ & $\mathrm{Li}$ & $\mathrm{NI}$ & $\mathrm{L}$ & $\begin{array}{l}\text { Decoction, } \\
\text { infusion }\end{array}$ & Cold, sinusitis & $\begin{array}{l}\text { Inhalation, } \\
\text { enema }\end{array}$ & 50 & $\begin{array}{l}\text { Two hands- } \\
\text { circumference of } \\
\text { leafy stem }\end{array}$ & 50 \\
\hline $\begin{array}{l}\text { Piliostigma thonningii (Schum.) } \\
\text { Milne-Redh. } \\
2210\end{array}$ & $\begin{array}{l}\text { Bag gnanga (M), } \\
\text { Nabali }(G)\end{array}$ & 4 & $\mathrm{Li}$ & $\mathrm{NI}$ & $\mathrm{L}$ & Decoction & $\begin{array}{l}\text { Diarrhea, } \\
\text { dysentery }\end{array}$ & Drink, bath & 40 & $\begin{array}{l}\text { Two hands- } \\
\text { circumference of } \\
\text { leafy stem }\end{array}$ & 50 \\
\hline $\begin{array}{l}\text { Pterocarpus erinaceus Poir. } \\
540\end{array}$ & Noèka (M) & 1 & $\mathrm{Li}$ & $\mathrm{NI}$ & $\mathrm{Re}, \mathrm{R}$ & Decoction & Diarrhea & Drink, bath & 13 & $\begin{array}{l}\text { One hand- } \\
\text { circumference of } \\
\text { stem bark }\end{array}$ & 50 \\
\hline
\end{tabular}




\begin{tabular}{|c|c|c|c|c|c|c|c|c|c|c|c|}
\hline $\begin{array}{l}\text { Tamarindus indica L. } \\
5326\end{array}$ & Pousga (M) & $1,2,3,5$ & $\mathrm{Li}$ & $\mathrm{NI}$ & $\mathrm{Sb}, \mathrm{S}$ & Decoction & $\begin{array}{l}\text { Stomachache, } \\
\text { wound healing }\end{array}$ & Drink, bath & 33 & $\begin{array}{l}\text { One hand- } \\
\text { circumference of } \\
\text { seed }\end{array}$ & 50 \\
\hline $\begin{array}{l}\text { Tephrosia bracteolata Guill. \& } \\
\text { Perr. } \\
2352\end{array}$ & Sanri (M) & 1 & $\mathrm{H}$ & $\mathrm{NI}$ & $\mathrm{L}$ & Decoction & $\begin{array}{l}\text { Dental pain, } \\
\text { headache }\end{array}$ & Bath & 3 & $\begin{array}{l}\text { One hand- } \\
\text { circumference of } \\
\text { the plant }\end{array}$ & 50 \\
\hline \multicolumn{12}{|l|}{ Lamiaceae } \\
\hline $\begin{array}{l}\text { Hyptis specigera Lam. } \\
2232\end{array}$ & $\begin{array}{l}\text { Rounroungu } \\
\text { (M) }\end{array}$ & 1 & $\mathrm{H}$ & $\mathrm{NI}$ & L & Decoction & $\begin{array}{l}\text { Malaria, dental } \\
\text { pain }\end{array}$ & $\begin{array}{l}\text { Bath, gargle, } \\
\text { enema }\end{array}$ & 10 & $\begin{array}{l}\text { One hand- } \\
\text { circumference of } \\
\text { the plant }\end{array}$ & 50 \\
\hline $\begin{array}{l}\text { Leonotis nepetaefolia (L.) R.Br. } \\
145\end{array}$ & $\begin{array}{l}\text { Barakourouni } \\
\text { (M) }\end{array}$ & 5 & $\mathrm{H}$ & $\mathrm{NI}$ & 1 & Decoction & Cold, sinusitis & Bath & 20 & $\begin{array}{l}\text { One hand- } \\
\text { circumference of } \\
\text { the plant }\end{array}$ & 50 \\
\hline $\begin{array}{l}\text { Ocimum americanum L. } \\
145\end{array}$ & Yulu-yuga (M) & 1,2 & $\mathrm{H}$ & $\mathrm{NI}$ & L, Sb, FI & $\begin{array}{l}\text { Decoction, } \\
\text { infusion }\end{array}$ & $\begin{array}{l}\text { Indigestion, } \\
\text { diarrhea, } \\
\text { rheumatism }\end{array}$ & Bath, inhalation & 40 & $\begin{array}{l}\text { One hand- } \\
\text { circumference of } \\
\text { the plant }\end{array}$ & 50 \\
\hline $\begin{array}{l}\text { Ocimum basilicum L. } \\
779\end{array}$ & $\begin{array}{l}\text { Yulin-gnuuga } \\
\text { (M) }\end{array}$ & 1,2 & $\mathrm{H}$ & $\mathrm{NI}$ & L & Decoction & Children fears & Bath & 33 & $\begin{array}{l}\text { One hand- } \\
\text { circumference of } \\
\text { the plant }\end{array}$ & 50 \\
\hline $\begin{array}{l}\text { Vitex cuneata Schumach. \& } \\
\text { Thonn. } \\
6190\end{array}$ & Andga (M) & 1 & $\mathrm{Li}$ & $\mathrm{NI}$ & $\mathrm{L}$ & Decoction & Diarrhea & Drink & 3 & $\begin{array}{l}\text { Two hands- } \\
\text { circumference of } \\
\text { leafy stem }\end{array}$ & 50 \\
\hline $\begin{array}{l}\text { Vitex doniana Sweet } \\
6189\end{array}$ & $\begin{array}{l}\text { Koto (D), Anda } \\
\text { (M) }\end{array}$ & 2 & $\mathrm{Li}$ & $\mathrm{NI}$ & $\mathrm{L}$ & Decoction & Hypertension & Drink & 3 & $\begin{array}{l}\text { Two hands- } \\
\text { circumference of } \\
\text { leafy }\end{array}$ & 50 \\
\hline \multicolumn{12}{|l|}{ Lauraceae } \\
\hline $\begin{array}{l}\text { Cassytha filiformis } \mathrm{L} . \\
586\end{array}$ & $\begin{array}{l}\text { Naam-kaboul } \\
\text { (M), Sanguidjo } \\
\text { (D) }\end{array}$ & $1,2,6$ & $H / L a$ & $\mathrm{NI}$ & $\mathrm{L}, \mathrm{Sb}$ & $\begin{array}{l}\text { Decoction, } \\
\text { infusion }\end{array}$ & Stomachache & Drink, bath & 50 & $\begin{array}{l}\text { One hand- } \\
\text { circumference of } \\
\text { the plant }\end{array}$ & 50 \\
\hline \multicolumn{12}{|l|}{ Loranthaceae } \\
\hline $\begin{array}{l}\text { Tapinanthus globiferus (A. Rich.) } \\
\text { Van Tiegh } \\
2875\end{array}$ & Soto (Fu) & 6 & Li, Pp & $\mathrm{NI}$ & $\mathrm{L}, \mathrm{Sb}$ & Decoction & Diarrhea & Bath & 7 & $\begin{array}{l}\text { One hand- } \\
\text { circumference of } \\
\text { leafy stem }\end{array}$ & 50 \\
\hline \multicolumn{12}{|l|}{ Lythraceae } \\
\hline $\begin{array}{l}\text { Lawsonia inermis L. } \\
3012\end{array}$ & Lallé (M) & $1,2,3,6$ & $\mathrm{Li}$ & $\mathrm{NI}$ & $\mathrm{Sb}$ & Decoction & Kidney & Purgative & 3 & $\begin{array}{l}\text { One hand- } \\
\text { circumference of } \\
\text { leafy stem }\end{array}$ & 50 \\
\hline \multicolumn{12}{|l|}{ Malvaceae } \\
\hline $\begin{array}{l}\text { Grewia mollis Juss. } \\
287\end{array}$ & $\begin{array}{l}\text { Mounou } \\
\text { mouka(M) }\end{array}$ & 3 & $\mathrm{Li}$ & $\mathrm{NI}$ & $\mathrm{Sb}$ & Decoction & $\begin{array}{l}\text { Growth } \\
\text { retardation }\end{array}$ & Drink, purgative & 3 & $\begin{array}{l}\text { One hand- } \\
\text { circumference of } \\
\text { leafy stem }\end{array}$ & 50 \\
\hline
\end{tabular}




\begin{tabular}{|c|c|c|c|c|c|c|c|c|c|c|c|}
\hline $\begin{array}{l}\text { Waltheria indica } \mathrm{L} . \\
5910\end{array}$ & $\begin{array}{l}\text { Yaryâamdém } \\
\text { (M) }\end{array}$ & $1,3,5$ & $\mathrm{H}$ & $\mathrm{NI}$ & $\mathrm{L}, \mathrm{R}$ & $\begin{array}{l}\text { Decoction, } \\
\text { trituration }\end{array}$ & $\begin{array}{l}\text { Growth } \\
\text { retardation }\end{array}$ & Bath, application & 50 & $\begin{array}{l}\text { One hand- } \\
\text { circumference of } \\
\text { the plant }\end{array}$ & 50 \\
\hline $\begin{array}{l}\text { Sida alba L. } \\
5878\end{array}$ & Zoaaga (M) & 3 & $\mathrm{H}$ & $\mathrm{NI}$ & $L$ & Decoction & Pitting edema & Enema & 7 & $\begin{array}{l}\text { One hand- } \\
\text { circumference of } \\
\text { plant }\end{array}$ & 50 \\
\hline $\begin{array}{l}\text { Wissadula amplissima (L.) R. E. } \\
\text { Fries. var. rostrata (Sch. \& Th.) } \\
\text { R.E. Fries. } \\
47\end{array}$ & $\begin{array}{l}\text { Gomtilaon-go } \\
\text { (M) }\end{array}$ & 1,3 & $\mathrm{H}$ & $\mathrm{NI}$ & $\mathrm{R}$ & Decoction & Cold, sinusitis & Bath & 13 & $\begin{array}{l}\text { One hand- } \\
\text { circumference of } \\
\text { the plant }\end{array}$ & 50 \\
\hline \multicolumn{12}{|l|}{ Meliaceae } \\
\hline $\begin{array}{l}\text { Khaya senegalensis (Desr.) A. } \\
\text { Juss. } \\
303\end{array}$ & Kuka (M) & 3 & $\mathrm{Li}$ & $\mathrm{NI}$ & $\mathrm{L}, \mathrm{Sb}$ & $\begin{array}{l}\text { Decoction, } \\
\text { maceration }\end{array}$ & Malaria & Drink, bath & 10 & $\begin{array}{l}\text { One hand- } \\
\text { circumference of } \\
\text { leafy stem }\end{array}$ & 50 \\
\hline $\begin{array}{l}\text { Pseudocedrela kotschyi } \\
\text { (Schweinf.) Harms } \\
72\end{array}$ & Sigdre (M) & 2,3 & $\mathrm{Li}$ & $\mathrm{Li}$ & $L, R$ & $\begin{array}{l}\text { Decoction, } \\
\text { calcination }\end{array}$ & $\begin{array}{l}\text { Hemorrhoids, } \\
\text { tiredness, tooth } \\
\text { pain }\end{array}$ & $\begin{array}{l}\text { Drink, massage, } \\
\text { brush }\end{array}$ & 40 & $\begin{array}{l}\text { One hand- } \\
\text { circumference of } \\
\text { leafy stem }\end{array}$ & 50 \\
\hline $\begin{array}{l}\text { Trichilia emetica Vahl } \\
2577\end{array}$ & $\begin{array}{l}\text { Sula fizân (D), } \\
\text { Kinkirs-taanga } \\
\text { (M) }\end{array}$ & 2,6 & $\mathrm{Li}$ & $\mathrm{NI}$ & $\mathrm{L}, \mathrm{Sb}$ & $\begin{array}{l}\text { Decoction, } \\
\text { trituration }\end{array}$ & $\begin{array}{l}\text { Hemorrhoids, } \\
\text { malaria }\end{array}$ & Drink & 10 & $\begin{array}{l}\text { Two hand- } \\
\text { circumference of } \\
\text { leafy stem }\end{array}$ & 100 \\
\hline \multicolumn{12}{|l|}{ Moraceae } \\
\hline $\begin{array}{l}\text { Ficus gnaphalocarpa (Miq.) } \\
\text { Steud. ex A. Rich. } \\
5584\end{array}$ & $\begin{array}{l}\text { Kamsogo (M), } \\
\text { Kankoabou(G) }\end{array}$ & 2,4 & $\mathrm{Li}$ & $\mathrm{Li}$ & $\mathrm{L}$ & Decoction & Malaria & Bath & 3 & $\begin{array}{l}\text { Two hands- } \\
\text { circumference of } \\
\text { leaf }\end{array}$ & 100 \\
\hline $\begin{array}{l}\text { Ficus platyphylla Del. } \\
8283\end{array}$ & $\begin{array}{l}\text { Kansaogo (M), } \\
\text { Kankanga (M) }\end{array}$ & 3 & $\mathrm{Li}$ & $\mathrm{NI}$ & L & Decoction & $\begin{array}{l}\text { Stomachache, } \\
\text { malaria }\end{array}$ & Drink, bath & 3 & $\begin{array}{l}\text { Two hands- } \\
\text { circumference of } \\
\text { leaf }\end{array}$ & 100 \\
\hline \multicolumn{12}{|l|}{ Moringaceae } \\
\hline $\begin{array}{l}\text { Moringa oleiffera Lam. } \\
7071\end{array}$ & Arzan tiiga (M) & 2 & $\mathrm{Li} / \mathrm{Ga}$ & $\mathrm{NI}$ & $\begin{array}{l}\mathrm{L}, \mathrm{R}, \mathrm{Sb}, \\
\mathrm{G}\end{array}$ & $\begin{array}{l}\text { Decoction, } \\
\text { infusion, } \\
\text { trituration }\end{array}$ & $\begin{array}{l}\text { Dysentery, } \\
\text { malaria, } \\
\text { stomachache, } \\
\text { general fatigue, } \\
\text { hypertension }\end{array}$ & Drink & 7 & $\begin{array}{l}\text { Two hands- } \\
\text { circumference of } \\
\text { leaf }\end{array}$ & 100 \\
\hline \multicolumn{12}{|l|}{ Ochnaceae } \\
\hline $\begin{array}{l}\text { Lophira lanceolata Van Tiegh. ex } \\
\text { Keay } \\
738\end{array}$ & $\begin{array}{l}\text { Mana (D), } \\
\text { Malanga (Fu) }\end{array}$ & 6 & $\mathrm{Li}$ & $\mathrm{NI}$ & $\mathrm{L}$ & Trituration & Skin disorders & Application & 3 & $\begin{array}{l}\text { Two hands- } \\
\text { circumference of } \\
\text { stem bark }\end{array}$ & 50 \\
\hline
\end{tabular}




\begin{tabular}{|c|c|c|c|c|c|c|c|c|c|c|c|}
\hline \multicolumn{12}{|l|}{ Olacaceae } \\
\hline $\begin{array}{l}\text { Ximenia americana L. } \\
8203 \mathrm{~N} 360\end{array}$ & Lenga (M) & 2,3 & $\mathrm{Li}$ & $\mathrm{NI}$ & $\mathrm{Sb}$ & Trituration & $\begin{array}{l}\text { Stomachache, } \\
\text { wound healing }\end{array}$ & Drink, bath & 10 & $\begin{array}{l}\text { One hand- } \\
\text { circumference of } \\
\text { stem bark }\end{array}$ & 50 \\
\hline \multicolumn{12}{|l|}{ Phyllantaceae } \\
\hline $\begin{array}{l}\text { Phyllanthus amarus Schum. \& } \\
\text { Thonn. } \\
6134\end{array}$ & Woompon-ré (M) & 1 & $\mathrm{H}$ & $\mathrm{NI}$ & L, Sb R & $\begin{array}{l}\text { Decoction, } \\
\text { Cataplasm, } \\
\text { infusion }\end{array}$ & $\begin{array}{l}\text { Hemorrhoids, } \\
\text { diarrhea, gout, } \\
\text { stomachache, } \\
\text { liver diseases }\end{array}$ & $\begin{array}{l}\text { Drink, bath, } \\
\text { purgative }\end{array}$ & 17 & $\begin{array}{l}\text { One hand- } \\
\text { circumference of } \\
\text { the plant }\end{array}$ & 50 \\
\hline \multicolumn{12}{|l|}{ Plantaginaceae } \\
\hline $\begin{array}{l}\text { Scoparia dulcis } \mathrm{L} \text {. } \\
3163\end{array}$ & $\begin{array}{l}\text { Tim tim }(D), \\
\text { Kafré-mandé (M) }\end{array}$ & 2,5 & $\mathrm{H}$ & $\mathrm{NI}$ & $\mathrm{L}, \mathrm{Sb}, \mathrm{R}$ & Decoction & $\begin{array}{l}\text { Cough, } \\
\text { hemorrhoid, } \\
\text { syphilis }\end{array}$ & Drink & 17 & $\begin{array}{l}\text { One hand- } \\
\text { circumference of } \\
\text { the plant }\end{array}$ & 50 \\
\hline \multicolumn{12}{|l|}{ Poaceae } \\
\hline $\begin{array}{l}\text { Andropogon gayanus Kunth } \\
20\end{array}$ & $\begin{array}{l}\text { Pittou (M), Waga } \\
\text { ou Karsabiin (D) }\end{array}$ & 2 & $\begin{array}{l}\mathrm{H} \\
/ \mathrm{F}\end{array}$ & $\mathrm{NI}$ & L & Decoction & Cough, malaria & Drink & 10 & $\begin{array}{l}\text { One hand- } \\
\text { circumference of } \\
\text { the plant }\end{array}$ & 100 \\
\hline $\begin{array}{l}\text { Bambusa vulgaris Schrad.ex } \\
\text { J.C. Wendl. } \\
3025\end{array}$ & $\begin{array}{l}\text { Nasar-tanwiiga } \\
\text { (M) }\end{array}$ & 2 & $\mathrm{H}$ & $\mathrm{NI}$ & $\mathrm{L}$ & Decoction & Malaria & Bath & 3 & $\begin{array}{l}\text { One hand- } \\
\text { circumference of } \\
\text { stem bark }\end{array}$ & 50 \\
\hline $\begin{array}{l}\text { Cymbopogon citratus (DC.) } \\
\text { Stapf } \\
3011\end{array}$ & Citronnelle (Fu) & 4 & $\mathrm{H} / \mathrm{Ga}$ & $\mathrm{NI}$ & L & Decoction & $\begin{array}{l}\text { Cold, sinusitis, } \\
\text { general fatigue }\end{array}$ & Drink & 3 & $\begin{array}{l}\text { One hand- } \\
\text { circumference of } \\
\text { the plant }\end{array}$ & 50 \\
\hline $\begin{array}{l}\text { Cymbopogon giganteus Chiov. } \\
8377\end{array}$ & Kuwere (M) & 2 & $\mathrm{H}$ & $\mathrm{NI}$ & $L, R$ & Decoction & $\begin{array}{l}\text { Prostate } \\
\text { diseases }\end{array}$ & Drink & 3 & $\begin{array}{l}\text { One hand- } \\
\text { circumference of } \\
\text { the plant }\end{array}$ & 50 \\
\hline $\begin{array}{l}\text { Cymbopogon schoenanthus (L.) } \\
\text { Spreng. } \\
5969\end{array}$ & Saabi (D) & 2 & $\mathrm{H}$ & $\mathrm{NI}$ & $L, S b, R$ & Trituration & Skin disorders & Application & 3 & $\begin{array}{l}\text { One hand- } \\
\text { circumference of } \\
\text { the plant }\end{array}$ & 50 \\
\hline \multicolumn{12}{|l|}{ Polygalaceae } \\
\hline $\begin{array}{l}\text { Securidaca longipedunculata } \\
\text { Fres. } \\
862\end{array}$ & Pelga (M) & 2,3 & $\mathrm{Li}$ & $\mathrm{NI}$ & $\mathrm{R}$ & Infusion & $\begin{array}{l}\text { Ulcer, liver } \\
\text { diseases, } \\
\text { respiratory } \\
\text { diseases }\end{array}$ & Enema, inhalation & 10 & $\begin{array}{l}\text { One hand- } \\
\text { circumference of } \\
\text { root }\end{array}$ & 300 \\
\hline \multicolumn{12}{|l|}{ Rubiaceae } \\
\hline $\begin{array}{l}\text { Crossopteryx } \\
\text { febrifuga (Afzel. ex G. Don) } \\
\text { Benth. } \\
15\end{array}$ & $\begin{array}{l}\text { Kumbr-wâaga } \\
\text { (M) }\end{array}$ & 4 & $\mathrm{Li}$ & $\mathrm{Li}$ & $\begin{array}{l}\text { L, Sb } \\
\mathrm{Fr}\end{array}$ & Decoction & Tonic & Drink & 3 & $\begin{array}{l}\text { One hand- } \\
\text { circumference of } \\
\text { leafy stem }\end{array}$ & 50 \\
\hline
\end{tabular}




\begin{tabular}{|c|c|c|c|c|c|c|c|c|c|c|c|}
\hline $\begin{array}{l}\text { Fadogia agrestis Schweinf. ex } \\
\text { Hiern } \\
286\end{array}$ & Bit koon-bré (M) & 1 & $\mathrm{Li}$ & $\mathrm{NI}$ & L, Sb & Decoction & Rheumatism & Bath & 17 & $\begin{array}{l}\text { One hand- } \\
\text { circumference of } \\
\text { stem bark }\end{array}$ & 50 \\
\hline $\begin{array}{l}\text { Feretia apodenthera Del. } \\
5374\end{array}$ & Mounouna (M) & 3,5 & $\mathrm{Li}$ & $\mathrm{NI}$ & L & Decoction & Stomachache & Drink & 3 & $\begin{array}{l}\text { Two hands- } \\
\text { circumference of } \\
\text { leafy stem }\end{array}$ & 50 \\
\hline $\begin{array}{l}\text { Gardenia erubescens Stapf \& } \\
\text { Hutch. } \\
2\end{array}$ & Susuba (M) & 4 & $\mathrm{Li}$ & $\mathrm{NI}$ & L, Sb & Decoction & $\begin{array}{l}\text { Healing of } \\
\text { umbilical cord } \\
\text { wounds }\end{array}$ & Enema & 7 & $\begin{array}{l}\text { One hand- } \\
\text { circumference of } \\
\text { leafy stem }\end{array}$ & 50 \\
\hline $\begin{array}{l}\text { Gardenia sokotensis Stapf \& } \\
\text { Hutch. } \\
8211 \text { N360 }\end{array}$ & $\begin{array}{l}\text { Tang-ra-kwenga } \\
\text { (M), } \\
\text { Toukôrôgoulé } \\
\text { (D) }\end{array}$ & $1,3,5$ & $\mathrm{Li}$ & $\mathrm{NI}$ & L & Decoction & $\begin{array}{l}\text { Tonic, malaria, } \\
\text { wound healing, } \\
\text { stomachache }\end{array}$ & Drink, bath & 67 & $\begin{array}{l}\text { One hand- } \\
\text { circumference of } \\
\text { leafy stem }\end{array}$ & 50 \\
\hline $\begin{array}{l}\text { Gardenia ternifolia Schum. \& } \\
\text { Thonn. } \\
2018\end{array}$ & $\begin{array}{l}\text { Râmbrezûnga } \\
\text { (M) }\end{array}$ & 1 & $\mathrm{Li}$ & $\mathrm{NI}$ & $\mathrm{Fr}$ & Decoction & Tonic & Bath & 10 & $\begin{array}{l}\text { One hand- } \\
\text { circumference of } \\
\text { fruit }\end{array}$ & 50 \\
\hline $\begin{array}{l}\text { Mitragyna inermis (Willd.) } \\
\text { Kuntze } \\
823\end{array}$ & Yilga (M) & 2,3 & $\mathrm{Li} / \mathrm{Cw}$ & $\mathrm{Li} / \mathrm{Cw}$ & $\mathrm{L}, \mathrm{Sb}$ & $\begin{array}{l}\text { Decoction, } \\
\text { infusion }\end{array}$ & $\begin{array}{l}\text { Malaria, } \\
\text { tiredness, } \\
\text { hypertension }\end{array}$ & Drink & 50 & $\begin{array}{l}\text { Two hand- } \\
\text { circumference of } \\
\text { fruit }\end{array}$ & 50 \\
\hline $\begin{array}{l}\text { Nauclea latifolia Smith } \\
2858\end{array}$ & Gouinga (M) & 2 & $\mathrm{Li}$ & $\mathrm{NI}$ & L,R & $\begin{array}{l}\text { Decoction, } \\
\text { infusion }\end{array}$ & $\begin{array}{l}\text { Wound healing, } \\
\text { stomachache }\end{array}$ & Drink, bath & 40 & $\begin{array}{l}\text { Two hand- } \\
\text { circumference of } \\
\text { fruit }\end{array}$ & 100 \\
\hline $\begin{array}{l}\text { Sarcocephalus latifolius (Smith) } \\
\text { Bruce } \\
2059\end{array}$ & Goinga(M) & 1 & $\mathrm{Li}$ & $\mathrm{NI}$ & $\mathrm{Sb}$ & Decoction & $\begin{array}{l}\text { Wound healing, } \\
\text { stomachache }\end{array}$ & Drink, bath & 50 & $\begin{array}{l}\text { One hand- } \\
\text { circumference of } \\
\text { stem bark }\end{array}$ & 50 \\
\hline \multicolumn{12}{|l|}{ Rutaceae } \\
\hline $\begin{array}{l}\text { Zanthoxylum zanthoxyloides } \\
\text { (Lam.) Zepern. \& Timler } \\
3061\end{array}$ & Rapèka (M) & 1 & $\mathrm{Li}$ & $\mathrm{NI}$ & $S b, R$ & Decoction & $\begin{array}{l}\text { Stomachache, } \\
\text { dental pain, } \\
\text { sickle-cell } \\
\text { disease }\end{array}$ & $\begin{array}{l}\text { Drink, bath, } \\
\text { toothbrush }\end{array}$ & 17 & $\begin{array}{l}\text { One hand- } \\
\text { circumference of } \\
\text { root bark }\end{array}$ & 200 \\
\hline \multicolumn{12}{|l|}{ Sapindaceae } \\
\hline $\begin{array}{l}\text { Paullinia pinnata L. } \\
2118\end{array}$ & $\begin{array}{l}\text { Nusanu (M), } \\
\text { Brodinburu (D) }\end{array}$ & $1,2,5,6$ & $\mathrm{Li}$ & $\mathrm{Li}$ & L, Sb, R & Decoction & $\begin{array}{l}\text { malaria, general } \\
\text { fatigue, } \\
\text { stomachache }\end{array}$ & Drink, Jbath & 67 & $\begin{array}{l}\text { One hand- } \\
\text { circumference of } \\
\text { leafy stem }\end{array}$ & 200 \\
\hline \multicolumn{12}{|l|}{ Sapotaceae } \\
\hline $\begin{array}{l}\text { Vitellaria paradoxa Gaertn. f. } \\
4683\end{array}$ & Taanga (M) & $1,2,3$ & $\mathrm{Li}$ & $\mathrm{NI}$ & $\mathrm{Sb}$ & $\begin{array}{l}\text { Decoction, } \\
\text { trituration }\end{array}$ & $\begin{array}{l}\text { Cold, sinusitis, } \\
\text { wound healing, } \\
\text { stomachache } \\
\text { hemorrhoids }\end{array}$ & $\begin{array}{l}\text { Drink, bath, } \\
\text { inhalation }\end{array}$ & 67 & $\begin{array}{l}\text { One hand- } \\
\text { circumference of } \\
\text { stem bark }\end{array}$ & 50 \\
\hline
\end{tabular}




\begin{tabular}{|c|c|c|c|c|c|c|c|c|c|c|c|}
\hline Solanaceae & & & & & & & & & & & \\
\hline $\begin{array}{l}\text { Physalis angulata L. } \\
642\end{array}$ & $\begin{array}{l}\text { Poapoa raaga } \\
\text { (M) }\end{array}$ & 1 & $\mathrm{H}$ & $\mathrm{NI}$ & L & Trituration & Skin disorders & Application & 3 & $\begin{array}{l}\text { One hand- } \\
\text { circumference of } \\
\text { the plant }\end{array}$ & 50 \\
\hline \multicolumn{12}{|l|}{ Verbenaceae } \\
\hline $\begin{array}{l}\text { Lantana camara L. } \\
2096\end{array}$ & $\begin{array}{l}\text { Nasar luili sibi } \\
\text { (M) }\end{array}$ & 1,2 & $\mathrm{H}$ & $\mathrm{NI}$ & $L, S b, R$ & $\begin{array}{l}\text { Decoction, } \\
\text { infusion }\end{array}$ & Hypertension & Drink & 3 & $\begin{array}{l}\text { One hand- } \\
\text { circumference of } \\
\text { the plant }\end{array}$ & 50 \\
\hline $\begin{array}{l}\text { Tectonia grandis L.f. } \\
3023\end{array}$ & Teck (F) & 1 & $\mathrm{NI}$ & $\mathrm{Li}$ & L & $\begin{array}{l}\text { Decoction, } \\
\text { maceration }\end{array}$ & Ulcer & Drink & 3 & $\begin{array}{l}\text { Two hands- } \\
\text { circumference of } \\
\text { leaf }\end{array}$ & 50 \\
\hline \multicolumn{12}{|l|}{ Vitaceae } \\
\hline $\begin{array}{l}\text { Cissus quadrangularius } \mathrm{L} \text {. } \\
4132 \mathrm{~A}\end{array}$ & Kilounoarou (G) & 4 & Li/La & $\mathrm{NI}$ & $\mathrm{Sb}$ & Cataplasm & Tiredness & Massage & 3 & $\begin{array}{l}\text { One hand- } \\
\text { circumference of } \\
\text { stem bark }\end{array}$ & 50 \\
\hline \multicolumn{12}{|l|}{ Zygophyllaceae } \\
\hline $\begin{array}{l}\text { Balanites aegyptiaca (L.) Del. } \\
5208\end{array}$ & Kyegelega (M) & 1 & $\mathrm{Li}$ & $\mathrm{NI}$ & $\mathrm{Fr}, \mathrm{Sb}$ & Decoction & $\begin{array}{l}\text { Stomachache, } \\
\text { hemorrhoids }\end{array}$ & Drink & 33 & $\begin{array}{l}\text { One hand- } \\
\text { circumference of } \\
\text { leafy stem }\end{array}$ & 50 \\
\hline
\end{tabular}

\section{Legend:}

Local name: D Dioula, G Gourmantché, Gsi Gourounsi, F French, M Mooré, Fu Fulfudé.

Provenance: Li ligneous, H Herbaceous, La Liana, Pp Parasitic plant, F Field, Ga Garden, He Hedgerow, Cw Coast watercourse, NI: No Information.

Part used: L Leaf, Sb Stem bark, B Bark, R Root, S Seed, G Gum, Fr Fruit, I Inflorescence, Sa Sap, Re Resin, FI Flora

City: Ouagadougou (1), Bobo Dioulasso (2), Ouahigoua (3), Fada (4), Dedougou (5), Léo (6). 
Table 2. Plant parts sold in the market for ailment treatment

\begin{tabular}{lll}
\hline Plant part used & $\begin{array}{l}\text { No. of plants } \\
\text { parts } \\
(\mathrm{n}=162)\end{array}$ & $\begin{array}{l}\text { \% of plant } \\
\text { parts }\end{array}$ \\
\hline Leaves & 76 & 47.0 \\
Stem bark & 55 & 34.0 \\
Root & 28 & 17.2 \\
Inflorescence & 1 & 0.6 \\
Gum & 1 & 0.6 \\
Seed & 1 & 0.6 \\
\hline
\end{tabular}

The total number of species for calculation of percentages was 162
Table 3. Source of medicinal plants

\begin{tabular}{lcc}
\hline Habitat & $\begin{array}{l}\text { No. of } \\
\text { species }\end{array}$ & $\begin{array}{l}\text { \% of } \\
\text { species }\end{array}$ \\
\hline Savanna & 101 & 86.3 \\
Forest & 3 & 2.6 \\
Savanna and forest & 9 & 7.7 \\
Savanna and field & 1 & 0.9 \\
Savanna and & 3 & 2.6 \\
garden & & \\
\hline
\end{tabular}

The total number of species for calculation of percentages was 117

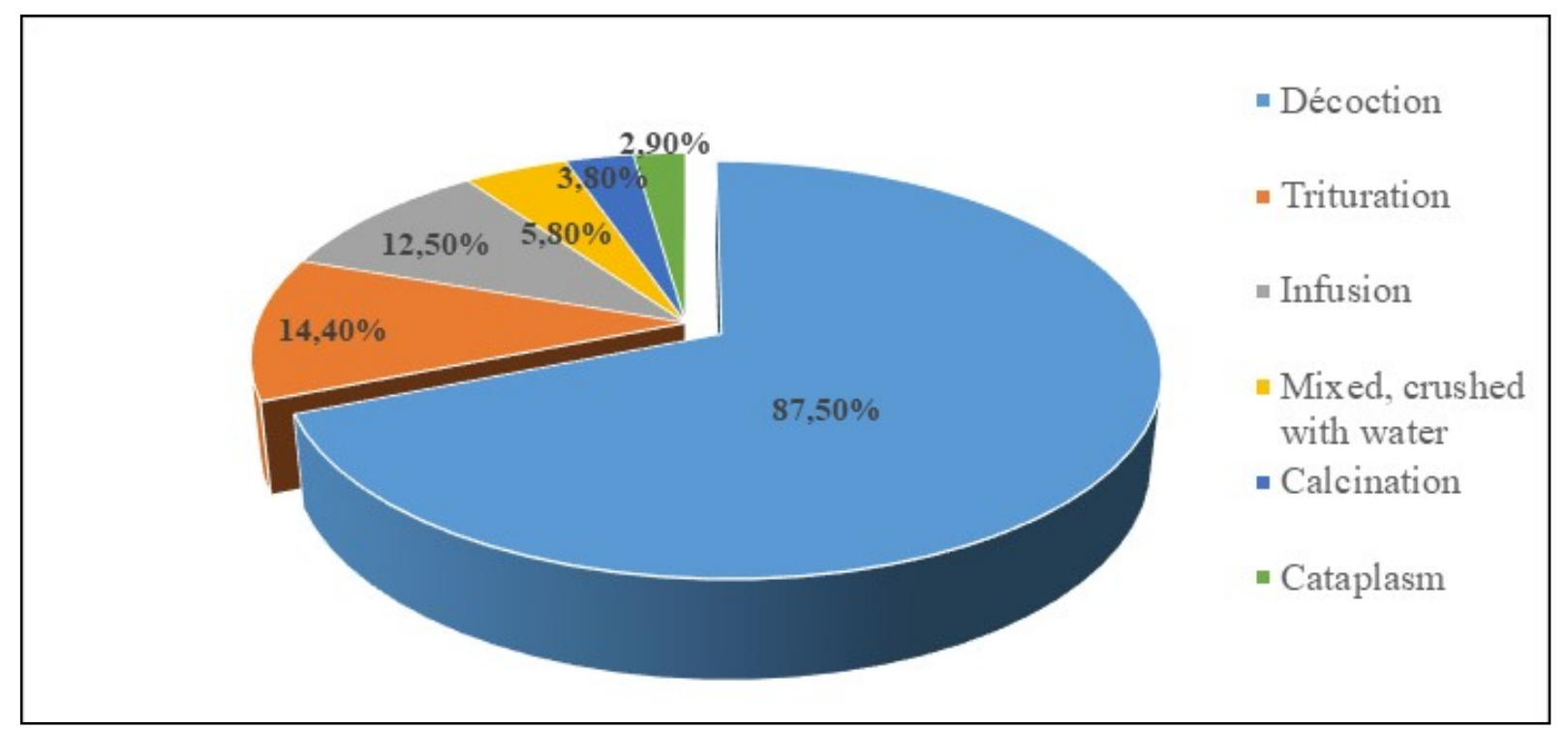

Figure 3. Percentage of species used for various recipe preparations. The total number of species for calculation of percentages was 104 . Some species were used for multiple recipe preparations.

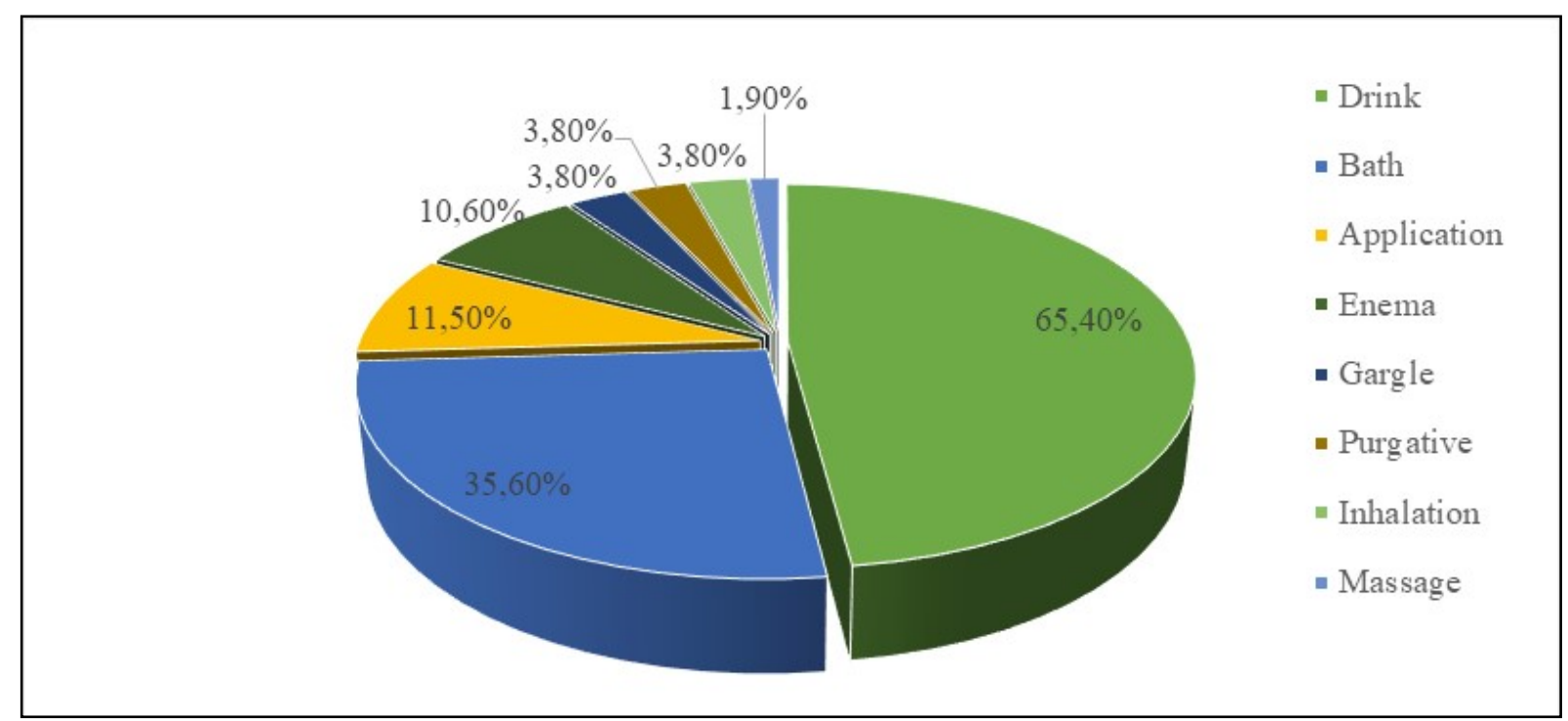

Figure 4. Percentage of species used for various administration modes. The total number of species for calculation of percentages was 104 . Some species were used for multiple administration modes. 
Table 4. Ailment categories (some species were used to treat multiple conditions)

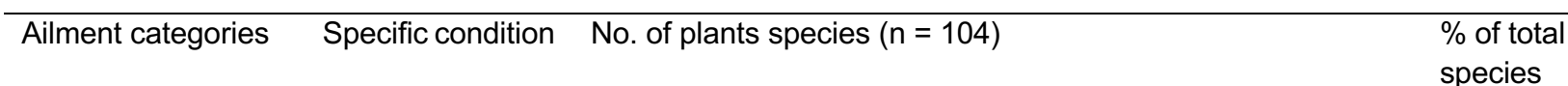

\begin{tabular}{lll}
\hline Gastrointestinal & Dysentery, ulcer, & Adansonia digitata (1), Afzelia africana (2), Alysicarpus \\
diseases & diarrhea, & glumaceus (3), Annona senegalensis (4), Baissea multiflora \\
& stomachache & (5), Balanites aegyptiaca (6), Boswellia dalzielii (7), Cassia
\end{tabular}
italica (8), Cassia nigricans (9), Cassia occidentalis (10), Cassia sieberiana (11), Cassia singueana (12), Cassytha filiformis (13), Cochlospermum tinctorium (14), Combretum adenogonium (15), Combretum glutinosum (16), Detarium microcarpum (17), Diospyros mespiliformis (18), Euphorbia hirta (19), Ficus platyphylla (20), Feretia apodenthera (21), Gardenia sokotensis (22), Guiera senegalensis (23), Heliotropium indicum (24), Lannea acida (25), Lepidagathis anobrya (26), Maytenus senegalensis (27), Moringa oleïfera (28), Nauclea latifolia (29), Ocimum americanum (30), Paullinia pinnata (31), Phyllanthus amarus (32), Piliostigma thonningii (33), Pteleopsis suberosa (34), Pterocarpus erinaceus (35), Pupalia lappacea (36), Saba senegalensis (37), Sarcocephalus latifolius (38), Sclerocarya birrea (39), Securidaca longipedunculata (40), Senegalia senegal (41), Tamarindus indica (42), Tapinanthus globiferus (43), Tectonia grandis (44), Terminalia avicennioïdes (45), Vachellia nilotica (46), Vitellaria paradoxa (47), Vitex cuneata (48), Ximenia Americana (49), Zanthoxylum zanthoxyloides (50)

\begin{tabular}{|c|c|c|c|}
\hline Malaria & - & $\begin{array}{l}\text { Andropogon gayanus (1), Annona senegalensis (2), } \\
\text { Bambusa vulgaris (3), Cochlospermum planchonii (4), } \\
\text { Cochlospermum tinctorium (5), Combretum micranthum (6), } \\
\text { Gardenia sokotensis (7), Guiera senegalensis (8), Cassia } \\
\text { sieberiana (9), Faidherbia albida (10), Ficus gnaphalocarpa } \\
\text { (11), Ficus platyphylla (12), Hyptis specigera (13), Khaya } \\
\text { senegalensis (14), Leptadenia hastate (15), Maytenus } \\
\text { senegalensis (16), Mitragyna inermis (17), Moringa oleïfera } \\
\text { (18), Paullinia pinnata (19), Trichilia emetica (20) }\end{array}$ & 19.2 \\
\hline $\begin{array}{l}\text { General pathology } \\
\text { symptoms }\end{array}$ & $\begin{array}{l}\text { Headache, } \\
\text { tiredness, cold, } \\
\text { insomnia, fatigue }\end{array}$ & $\begin{array}{l}\text { Annona senegalensis (1), Bauhinia rufescens (2), Cissus } \\
\text { quadrangularius (3), Combretum sericeum (4), Cymbopogon } \\
\text { citratus (5), Detarium microcarpum (6), Entada africana (7), } \\
\text { Guiera senegalensis (8), Leonotis nepetaefolia (9), Maytenus } \\
\text { senegalensis (10), Mitragyna inermis (11), Moringa oleïfera } \\
\text { (12), Paullinia pinnata (13), Piliostigma reticulatum (14), } \\
\text { Pseudocedrela kotschyi (15), Senegalia macrostachya (16), } \\
\text { Tephrosia bracteolate (17), Vitellaria paradoxa (18), } \\
\text { Wissadula amplissima (19) }\end{array}$ & 18.3 \\
\hline Dermatosis & $\begin{array}{l}\text { Skin disorders, } \\
\text { wound healing }\end{array}$ & $\begin{array}{l}\text { Annona senegalensis (1), Boswellia dalzielii (2), Cassia alata } \\
\text { (3), Cassia italica(4), Cassia nigricans (5), Diospyros } \\
\text { mespiliformis (6), Gardenia sokotensis (7), Lophira lanceolata } \\
\text { (8), Cymbopogon schoenanthus (9), Nauclea latifolia (10), } \\
\text { Parkia biglobosa (11), Physalis angulata (12), Pupalia } \\
\text { lappacea (13), Saba senegalensis (14), Sarcocephalus } \\
\text { latifolius (15), Vitellaria paradoxa (16), Tamarindus indica } \\
\text { (17), Ximenia americana (18) }\end{array}$ & 17.3 \\
\hline $\begin{array}{l}\text { Respiratory } \\
\text { diseases }\end{array}$ & $\begin{array}{l}\text { Respiratory } \\
\text { disorder, } \\
\text { sinusitis, cough }\end{array}$ & $\begin{array}{l}\text { Andropogon gayanus (1), Anogeissus leiocarpa (2), Bauhinia } \\
\text { rufescens (3), Burkea africana (4), Cymbopogon citratus (5), } \\
\text { Dichrostachys cinerea (6), Entada africana (7), Guiera } \\
\text { senegalensis (8), Euphorbia hirta (9), Hygrophila auriculata } \\
\text { (10), Leonotis nepetaefolia (11), Piliostigma reticulatum (12), }\end{array}$ & 17.3 \\
\hline
\end{tabular}




\begin{tabular}{|c|c|c|c|}
\hline & & $\begin{array}{l}\text { Pteleopsis suberosa (13), Scoparia dulcis (14), Securidaca } \\
\text { longipedunculata (15), Vachellia nilotica (16), Vitellaria } \\
\text { paradoxa (17), Wissadula amplissima (18) }\end{array}$ & \\
\hline Hemorrhoids & - & $\begin{array}{l}\text { Balanites aegyptiaca (1), Combretum paniculatum (2), Parkia } \\
\text { biglobosa (3), Phyllanthus amarus (4), Pseudocedrela } \\
\text { kotschyi (5), Pteleopsis suberosa (6), Scoparia dulcis (7), } \\
\text { Senegalia Senegal (8), Terminalia macroptera (9), Trichilia } \\
\text { emetica (10), Vachellia nilotica (11), Vitellaria paradoxa (12) }\end{array}$ & 11.5 \\
\hline $\begin{array}{l}\text { Internal organ } \\
\text { diseases }\end{array}$ & $\begin{array}{l}\text { Liver diseases, } \\
\text { kidney diseases }\end{array}$ & $\begin{array}{l}\text { Cassia alata (1), Cassia sieberiana (2), Chrysanthellum } \\
\text { americanum (3), Combretum glutinosum (4), Faidherbia } \\
\text { albida (5), Lawsonia inermis (6), Securidaca longipedunculata } \\
\text { (7), Phyllanthus amarus (8), Terminalia avicennioïdes (9) }\end{array}$ & 8.7 \\
\hline $\begin{array}{l}\text { Cardiovascular } \\
\text { diseases }\end{array}$ & $\begin{array}{l}\text { Heart aches, } \\
\text { control of } \\
\text { heartbeat, } \\
\text { hypertension }\end{array}$ & $\begin{array}{l}\text { Anacardium occidentale (1), Burkea africana (2), } \\
\text { Heliotropium indicum (3), Lantana camara (4), Mitragyna } \\
\text { inermis (5), Moringa oleïfera (6),Terminalia avicennioïdes (7), } \\
\text { Vitex doniana (8) }\end{array}$ & 7.7 \\
\hline $\begin{array}{l}\text { Infections (excl. } \\
\text { malaria) }\end{array}$ & $\begin{array}{l}\text { Dental infections, } \\
\text { eye infections }\end{array}$ & $\begin{array}{l}\text { Combretum aculeatum (1), Cynanchum viminale (2), } \\
\text { Maytenus senegalensis (3), Tephrosia bracteolata (4), Hyptis } \\
\text { specigera (5), Hyptis specigera (6) }\end{array}$ & 5.8 \\
\hline Child diseases & $\begin{array}{l}\text { Umbilical cord } \\
\text { treatment, } \\
\text { children fears, } \\
\text { growth } \\
\text { retardation }\end{array}$ & $\begin{array}{l}\text { Alternanthera nodiflora (1), Gardenia erubescens (2), Grewia } \\
\text { mollis (3), Lepidagathis anobrya (4), Ocimum basilicum (5), } \\
\text { Waltheria indica (6) }\end{array}$ & 5.8 \\
\hline Urogenital diseases & $\begin{array}{l}\text { Syphilis, } \\
\text { prostate, sexual } \\
\text { impotence }\end{array}$ & $\begin{array}{l}\text { Cassia sieberiana (1), Cymbopogon giganteus (2), Diospyros } \\
\text { mespiliformis (3), Leptadenia hastate (4), Scoparia dulcis (5) }\end{array}$ & 4.8 \\
\hline Arthritis & $\begin{array}{l}\text { Rheumatism and } \\
\text { gout }\end{array}$ & $\begin{array}{l}\text { Fadogia agrestis (1), Phyllanthus amarus (2), Ocimum } \\
\text { americanum (3) }\end{array}$ & 2.9 \\
\hline Blood diseases & $\begin{array}{l}\text { Sickle cell } \\
\text { disease, anemia }\end{array}$ & Hippocratea africana (1), Zanthoxylum zanthoxyloides (2) & 1.9 \\
\hline
\end{tabular}

\section{Fidelity Levels (FL) of plant species}

The FL values reflect the concordance between informants in using a particular plant species for treating of a specific disease in relation to the total numbers of use reports of that particular species for treating any disease. The highest fidelity level of all plant species was attributed to Gardenia sokotensis Stapf \& Hutch. ( $F L=80 \%$ ) used for treatment of malaria (Table 7). This was followed by $B$. rufescens for usage as tonic and Pteleopsis suberosa Engl. \& Diels for treatment of stomachache ( $F L=77 \%$ for both species). Two other species, C. micranthum and Maytensus senegalensis (Lam.) Exell exhibited also rather high-fidelity levels in treatment of malaria ( $F L=75 \%$ for both species).

\section{Presence of species in the markets and prices demanded}

The species most frequently offered in the stalls (present in $\geq 67 \%$ of all stalls) were Annona senegalensis Pers., G. sokotensis, Paullinia pinnata L., $P$. suberosa and $V$. paradoxa (Table 8). As prices of the medicinal plants varied somehow from one market to market, we give an average price estimate in Table 1 for a one hand-circumference quantity. For the majority of species prices were 50 FCFA for a bundle of one hand-circumference. Higher prices were demanded for some species which were rarely commercialized, like Chrysanthellum americanum (L.) Vatke, Securidaca longipedunculata Fres., and Zanthoxylum zanthoxyloides (Lam.) Zepern. \& Timler (up to 300 FCFA for a one handcircumference bundle). However, rarity is not the only condition for being a highly valued species, as found for $P$. pinnata which was offered in $67 \%$ of the markets and priced for 200 FCFA per bundle.

\section{Conservation status of species encountered in the markets}

For only $19 \%$ of the species encountered in the markets an estimation of the IUCN conservation status is available (IUCN 2018). Within the 20 most frequently commercialized species nine are qualified in the category Data Deficient, as there are currently not sufficient data for a precise evaluation of the extinction risk. Ten species have a stable population status and one ( $V$. paradoxa) is assessed by IUCN as vulnerable (Table 8). Three additional species found in the markets have a vulnerable status: Afzelia africana Smith ex Pers., Khaya senegalensis (Desr.) A. Juss. and Tapianthus globiferus (A.Rich.) 
Van Tiegh. These species were rarely present in the stalls (frequency of citation $\leq 10 \%$ ).

Table 5. Informant consensus factor (FIC) about ailment treatment by medicinal plants

\begin{tabular}{lccc}
\hline Ailment category & $\mathrm{N}_{\mathrm{ur}}$ & $\mathrm{N}$ & $\mathrm{FIC}$ \\
\hline Child diseases & 48 & 6 & 0.9 \\
Cardiovascular diseases & 38 & 8 & 0.8 \\
Respiratory diseases & 68 & 19 & 0.7 \\
Infectious diseases (excl. malaria) & 21 & 7 & 0.7 \\
General pathology symptoms & 71 & 21 & 0.7 \\
Arthritis & 8 & 3 & 0.7 \\
Dermatosis & 52 & 21 & 0.6 \\
Malaria & 45 & 19 & 0.6 \\
Gastrointestinal disease & 125 & 50 & 0.6 \\
Hemorrhoids & 26 & 11 & 0.6 \\
Blood diseases & 3 & 2 & 0.5 \\
Urogenital diseases & 8 & 5 & 0.4 \\
Internal organ diseases & 12 & 9 & 0.3 \\
\hline
\end{tabular}

$\mathrm{N}_{\mathrm{ur}}=$ Number of use reports by category from vendors in the markets. $\mathrm{N}=$ Number of species listed in each category from all vendors. FIC= Informant Consensus Factor

Table 6. Rank value assigned by key informants for the 10 preferred medicinal plants

\begin{tabular}{|c|c|c|c|c|c|c|c|c|c|c|c|c|c|}
\hline \multirow{2}{*}{$\begin{array}{l}\text { Medicinal } \\
\text { plant species }\end{array}$} & \multirow{2}{*}{$\begin{array}{l}\text { Part } \\
\text { used }\end{array}$} & \multirow{2}{*}{$\begin{array}{l}\text { Ailment / } \\
\text { Symptoms } \\
\text { treated }\end{array}$} & \multicolumn{9}{|c|}{ Key informants $(n=9)$} & \multirow{2}{*}{$\begin{array}{l}\text { Value } \\
190\end{array}$} & \multirow[t]{2}{*}{ Rank } \\
\hline & & & $A$ & $B$ & $\mathrm{C}$ & $\mathrm{D}$ & $E$ & $\mathrm{~F}$ & $\mathrm{G}$ & $\mathrm{H}$ & I & & \\
\hline $\begin{array}{l}\text { Combretum } \\
\text { micranthum }\end{array}$ & Leaf & Malaria & 10 & 8 & 9 & 10 & 8 & 10 & 8 & 9 & 10 & 82 & $1^{\text {st }}$ \\
\hline $\begin{array}{l}\text { Vitellaria } \\
\text { paradoxa }\end{array}$ & $\begin{array}{l}\text { Stem } \\
\text { bark }\end{array}$ & $\begin{array}{l}\text { Cold, sinusitis, } \\
\text { wound } \\
\text { healing, } \\
\text { hemorrhoids }\end{array}$ & 10 & 8 & 10 & 8 & 9 & 10 & 8 & 8 & 6 & 77 & $2^{\text {nd }}$ \\
\hline $\begin{array}{l}\text { Vachelia } \\
\text { nilotica }\end{array}$ & $\begin{array}{l}\text { Fruit, } \\
\text { stem } \\
\text { bark }\end{array}$ & $\begin{array}{c}\text { Stomachache, } \\
\text { hemorrhoids, } \\
\text { cough }\end{array}$ & 9 & 8 & 6 & 10 & 9 & 10 & 10 & 8 & 7 & 77 & $3^{\text {rd }}$ \\
\hline $\begin{array}{l}\text { Gardenia } \\
\text { sokotensis }\end{array}$ & Leaf & $\begin{array}{c}\text { Tonic, malaria, } \\
\text { wound } \\
\text { healing, } \\
\text { stomachache }\end{array}$ & 8 & 10 & 8 & 10 & 8 & 6 & 8 & 10 & 6 & 74 & $4^{\text {th }}$ \\
\hline $\begin{array}{l}\text { Maytenus } \\
\text { senegalensis }\end{array}$ & $\begin{array}{l}\text { Leaf, } \\
\text { root }\end{array}$ & $\begin{array}{c}\text { Malaria, } \\
\text { diarrhea, } \\
\text { dental pain, } \\
\text { headache }\end{array}$ & 10 & 8 & 10 & 7 & 9 & 8 & 5 & 6 & 10 & 73 & $5^{\text {th }}$ \\
\hline $\begin{array}{l}\text { Lepidagathis } \\
\text { anobrya }\end{array}$ & Leaf & $\begin{array}{c}\text { Growth } \\
\text { retardation, } \\
\text { stomachache }\end{array}$ & 8 & 9 & 10 & 6 & 8 & 7 & 10 & 5 & 6 & 69 & $6^{\text {th }}$ \\
\hline $\begin{array}{l}\text { Pteleopsis } \\
\text { suberosa }\end{array}$ & $\begin{array}{l}\text { Leaf, } \\
\text { stem } \\
\text { bark }\end{array}$ & $\begin{array}{c}\text { Dysentery, } \\
\text { stomachache, } \\
\text { cough, } \\
\text { hemorrhoids }\end{array}$ & 7 & 8 & 10 & 8 & 8 & 7 & 6 & 5 & 8 & 67 & $7^{\text {th }}$ \\
\hline $\begin{array}{l}\text { Paullinia } \\
\text { pinnata }\end{array}$ & $\begin{array}{l}\text { Leaf, } \\
\text { stem } \\
\text { bark, } \\
\text { root }\end{array}$ & $\begin{array}{c}\text { Malaria, } \\
\text { general } \\
\text { fatigue, } \\
\text { stomachache }\end{array}$ & 8 & 6 & 8 & 9 & 7 & 5 & 7 & 8 & 7 & 65 & $8^{\text {th }}$ \\
\hline $\begin{array}{l}\text { Bauhinia } \\
\text { rufescens }\end{array}$ & $\begin{array}{l}\text { Leaf, } \\
\text { stem }\end{array}$ & $\begin{array}{l}\text { Cold, sinusitis, } \\
\text { tonic }\end{array}$ & 8 & 10 & 9 & 5 & 6 & 5 & 8 & 7 & 6 & 64 & $9^{\text {th }}$ \\
\hline
\end{tabular}




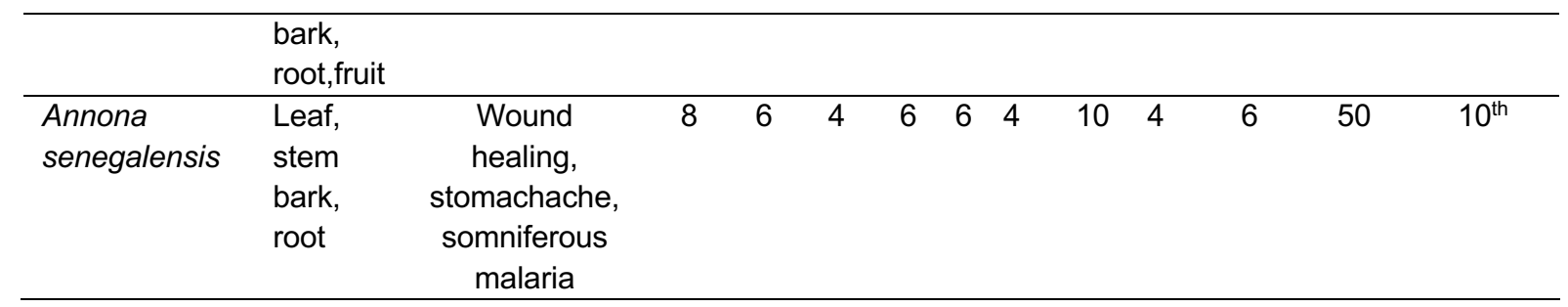

Ranks given to a medicinal plant are based on its efficacy in treating a special ailment according to the informant. Highest number (10) for a medicinal plant is given when informants considered the plant being most effective in healing an ailment and lowest (1) for the least effective.

Table 7. Fidelity levels of most common species used

\begin{tabular}{lllll}
\hline Medicinal plant species & Therapeutic use & $I_{P}$ & $I_{U}$ & $F L[\%]$ \\
\hline Gardenia sokotensis & Malaria & 16 & 20 & 80 \\
Bauhinia rufescens & Tonic & 20 & 26 & 77 \\
Pteleopsis suberosa & Stomachache & 10 & 13 & 77 \\
Combretum micranthum & Malaria & 15 & 20 & 75 \\
Maytenus senegalensis & Malaria & 18 & 24 & 75 \\
Vitellaria paradoxa & Sinusitis & 15 & 20 & 75 \\
Vachellia nilotica & Stomachache & 18 & 25 & 72 \\
Lepidagathis anobrya & Growth retardation & 11 & 16 & 69 \\
Paullinia pinnata & Stomachache & 10 & 20 & 50 \\
Annona senegalensis & Stomachache & 10 & 20 & 50 \\
\hline
\end{tabular}

Ip = Number of informants who suggested the use of a species for the same major ailment; lu = Total number of informants who mentioned the species for any use

\section{Discussion}

\section{Informant characteristics}

Women by far prevailed in the trade of medicinal plants in the studied markets in Burkina Faso. In Africa, this trade is commonly dominated by women (Van Andel et al. 2012). The average age of vendors of medicinal plants was about 54 years. This relatively high age may be attributed to two reasons: In Africa herbalists of higher age ( $>50$ ) are commonly estimated to have acquired more knowledge in the area of medicinal plants (Tugume et al. 2016). Until recently this knowledge has traditionally been passed to the young generation by oral communication, but nowadays younger people have little interest in learning and practicing this activity.

Most of the traditional healers were affiliated with an association in the field of medicinal plants. These associations possess a formal authorization from the government to exercise this activity.

\section{Diversity of medicinal plant species and plant parts used}

The high number of species recorded (104) proves that Burkina Faso has a diverse flora used as remedies against ailments and rich traditional knowledge on medicinal plants is still present in the community. In a similar investigation performed in another country of the Sahel zone, North Sudan, only 44 plant species could be identified for medicinal uses (Suleiman 2015). The most represented plant families in our study were Fabaceae, Combretaceae, and Rubiaceae. A high rate of utilization of these plant families has also been reported in other studies on medicinal plant use in Burkina Faso (Zerbo et al. 2011; Ky et al. 2009). Fabaceae was also the most represented plant family in an ethnobotanical study in Togo which highlights the importance of this family in traditional medicine (Agbodeka et al. 2015). The plant parts preferably used for medicinal recipes were leaves followed by barks and roots. In previous investigations in other regions of Burkina Faso (province of Kadiogo and Kouritenga), leaves were also the predominant plant parts collected (Tapsoba and Deschamps 2006; Nadembega et al. 2011). The high utilization rate of leaves could be attributed to the ease with which they can be obtained in large quantities compared to other plant parts and the abundance of bioactive compounds usually found in leaves (Tugume et al. 2016; Nadembega et al. 2011). Large quantities of roots and barks were also collected for recipe preparations. If not properly controlled, this destructive harvesting practice certainly threatens the survival of numerous medicinal plant species in the long term (Bhattarai 1997). 
Table 8. Frequency of citation of medicinal plant species most commonly encountered on market stalls and corresponding conservation status according to the IUCN Red List

\begin{tabular}{clcc}
\hline $\mathbf{N}^{\circ}$ & Medicinal plant species & $\begin{array}{c}\text { Frequency of citation } \\
(\%)\end{array}$ & $\begin{array}{c}\text { IUCN Conservation } \\
\text { status }\end{array}$ \\
\hline $\mathbf{1}$ & Annona senegalensis & 67 & LC \\
$\mathbf{2}$ & Gardenia sokotensis & 67 & DD \\
$\mathbf{3}$ & Paullinia pinnata & 67 & DD \\
$\mathbf{4}$ & Pteleopsis suberosa & 67 & LC \\
$\mathbf{5}$ & Vitellaria paradoxa & 67 & VU \\
$\mathbf{6}$ & Bauhinia rufescens & 50 & LC \\
$\mathbf{7}$ & Cassia sieberiana & 50 & LC \\
$\mathbf{8}$ & Cassytha filiformis & 50 & DD \\
$\mathbf{9}$ & Combretum aculeatum & 50 & LC \\
$\mathbf{1 0}$ & Combretum micranthum & 50 & LC \\
$\mathbf{1 1}$ & Guiera senegalensis & 50 & LC \\
$\mathbf{1 2}$ & Lepidagathis anobrya & 50 & DD \\
$\mathbf{1 3}$ & Maytenus senegalensis & 50 & DD \\
$\mathbf{1 4}$ & Mitragyna inermis & 50 & DD \\
$\mathbf{1 5}$ & Piliostigma reticulatum & 50 & LC \\
$\mathbf{1 6}$ & Saba senegalensis & 50 & LC \\
$\mathbf{1 7}$ & Sclerocarya birrea & 50 & DD \\
$\mathbf{1 8}$ & Terminalia avicennoïdes & 50 & DD \\
$\mathbf{1 9}$ & Vachellia nilotica var. adansonii & 50 & LC \\
$\mathbf{2 0}$ & Waltheria indica & 50 & DD \\
\hline
\end{tabular}

DD data deficient; LC least concern; VU vulnerable

As can be expected the most varied range of species was offered in the two largest cities of Burkina Faso, Ouagadougou and Bobo Dioulasso. Most of the species were found in at least two markets. The most unique medicinal plant panels with numerous plants not found in other markets were offered in the cities Fada N'Gourma and Léo. Whether this reflects specific cultural or social differences linked to these regions is beyond the scope of this study.

Herbal medicine preparation and administration Decoction is by far the most common method of preparation, as already described in previous ethnobotanical studies in Burkina Faso (Zerbo et al. 2011; Tapsoba and Deschamps 2006). Water is the cheapest and the most available solvent for rapid extraction of active ingredients, but some of the active metabolites of plants can be degraded by extraction performed at high temperatures (Kasali et al. 2014). Decoction by boiling or shaking herbals uses large quantities of plant material and may thus contribute to overexploitation of species (Tugume et al. 2016). Drinking was the main route of administration, which is common practice for treatment of ailments in families (Togola et al. 2005).

Quantitative analysis of the field study for highlighting the most important plants

One of the objectives of this investigation was to identify plants that are of particular importance for the treatment of certain diseases. For this, a set of ethnobotanical indices was used for quantitative analysis of the results of the structured interviews performed with the herbalists.

The factor of informant consensus (FIC value) is particularly useful for selecting the categories of diseases for which medicinal plants are traditionally used. By applying this tool, researchers can identify the main diseases in a community grouped by the categories for which medicinal plants are used. The highest FIC value was found for childhood illnesses, followed by cardiovascular diseases. The most cited species for the treatment of childhood diseases was the herbaceous plant, Lepidagathis anobrya (Acanthaceae). This species is particularly used for the cure of growth retardation and stomach pain in children. Ocimum basilicum (Lamiaceae), a globally estimated herb, was also often cited by the vendors for the treatment of children fears. Cardiovascular diseases come in second in the disease ranking. As a remedy against this ailment, the vendors particularly mentioned Mitragyna inermis as being highly effective against hypertension. These results are in line with those of Konkon et al. (2008) who demonstrated that the anti-hypertensive activity of $M$. inermis is due to the presence of triterpenes.

In addition to the factor of informant consensus, the fidelity level was applied to the data of our field study for selecting important individual plants. Using this 
tool, the species Gardenia sokotensis, Combretum micranthum and Maytenus senegalensis ranked as the best candidates for treatment of malaria. These three species were also among the favorites when we asked vendors to perform a preference ranking of the ten most valued plants in terms of frequency of use and effectiveness against malaria.

Thus, the combination of fidelity level and preference ranking highlights $G$. sokotensis, C. micranthum and $M$. senegalensis as particularly important species for treatment of malaria. G. sokotensis is described as containing multiple chemical compounds including saponosides, alkaloids, triterpens and sterols (Ouédraogo 1998). Previous studies have already described the anti-plasmodial efficacy of $G$. sokotensis and $M$. senegalensis (Ouédraogo 1998; Willcox et al. 2011). Extracts from C. micranthum have already been tested clinically and have demonstrated partial parasite clearance and very efficient fever clearance after seven days of treatment (Willcox et al. 2011). The high frequency of citation of these three species also demonstrates the high prevalence of malaria in Burkina Faso. According to WHO (2009) and several other studies (Ky et al. 2009; Zizka et al. 2015), malaria is a major threat to the population of Burkina Faso, with 3.5 million recorded cases in 2008 (thereof $50 \%$ of the children under 5 years).

The results of our field study highlight the value of traditional knowledge in detecting plant species containing pharmacological effective substances with potential to treat this important disease. We thus strongly encourage the development of plant material derived from $G$. sokotensis, $C$. micranthum and $M$. senegalensis into standardized phytotherapeutical drugs. This can be done quickly and cheaply and ensures the access to complementary medicine for the poorest people in remote areas. The use of alternative active substances might also delay the development of resistance to current standard drugs. As all three plants have a long tradition in the treatment of malaria by traditional healers in Burkina Faso and Mali (Zizka et al. 2015; Haidara et al. 2016), severe toxicological and safety issues are not to be expected.

In the present survey, combinations of plants for the treatment of diseases were not reported by the vendors. In contrast, treatment of several diseases with a single plant seems to be common practice. For example, Hippocratea africana (Wild.) Loes. or Vitex doniana Sweet were used to treat sickle-cell disease and hypertension, while Euphorbia hirta L. was used for the treatment of dysentery and respiratory troubles. Some medicinal plants were used to treat three or more types of diseases. An example for this is Mitragyna inermis, which by the informants was mainly mentioned for hypertension treatment but was also recorded to treat malaria, wounds and stomach aches. This is substantiated by several studies that have reported positive effects of $M$. inermis against stomachache, malaria, diabetes, dysentery, venereal diseases and mental disorders (Sourabie et al. 2013). According to Tugume et al. (2016), the use of a plant to treat several ailments is probably attributed to the presence of many metabolites in this plant and also to the observation that the same molecule can be active against different pathologies.

\section{Conservational aspects}

In Burkina Faso, most of the plants sold in the markets are collected in the savanna by traditional health practitioners, sellers or collectors. Whereas the traditional health practitioners are instructed to collect herbal material in a sustainable manner by government, numerous uncontrolled collectors represent a serious risk for medicinal plant species (Nadembega et al. 2011). This risk is also increased by the fact that most plant parts are collected from ligneous species in the savannas (Kristensen et al. 2003; Moyo et al. 2015). Savanna trees are often overexploited because of their multiple usages, not only for medicine products, but also for domestic energy, timber for construction, seeds for food preparation or fodder for animals. The Vitellaria paradoxa tree is such a multi-purpose species that was among the most cited species encountered in the markets and plant material was sold in large quantities in nearly $70 \%$ of the stalls at a relatively low price (one hand-circumference of stem bark for 50 FCFA). Unsustainable collection of the stem bark of this tree by local drug collectors could lead to the disappearance of this species in the savanna. In addition to the bark collected for preparation of remedies, the nuts of this tree are also intensively collected by local women to obtain oil used for food and for preparation of cosmetics products. This impacts the reproduction of the tree populations from seeds and thus further increases the extinction pressure on this species. It is classified by the IUCN as a vulnerable species. According to forest villagers and our own observations in the field, $V$. paradoxa is now in fact a very rare plant found in the savanna area. Some other species as Securidaca longipedunculata and Zanthoxylum zanthoxyloides are already diminished in their native environment (own observations). They were rarely offered in the stalls and their prices greatly exceeded the ones for readily available species converting them into a highly profitable natural resource. Three additional tree species which are assessed as vulnerable by IUCN (Afzelia africana, Tapianthus globiferus, Khaya 
senegalens) were sold at low prices, nevertheless they were quite rarely found in the markets. The stem bark and roots of $A$. africana are used in phytomedicine, a practice that particularly endangers this species. From $T$. globiferus and $K$. senegalensis leaves or stem bark are used for the preparation of extracts. The vulnerable status of Tapinanthus globiferus could be explained by the fact that it parasitizes on V. paradoxa (Ahamidé et al. 2017). For many other species, like Annona senegalensis, Bauhinia rufescens or Cassia occidentalis L. (for further species see Table 1), the stem bark, the roots and the leaves have been cited as a remedy for the same ailment. Sustainability of these species would be secured if harvesting of bark and root is avoided and instead harvesting of leaves would be promoted.

Unfortunately, medicinal plants are cultivated only in rare cases in home gardens, as hedges or in nurseries in Burkina Faso. This is underlined by our survey as we found very rarely cultivated plants in the stalls of the vendors. There are several reasons for this. Firstly, institutional support for the production and dissemination of approved plant material for cultivation is very limited (Cunningham 1997). Secondly, there is a common belief that cultivated plants do not contain the same composition and quantities of active ingredients as plants grown in their native environments. The rapid urbanization currently in progress in Burkina Faso will inevitably lead to an excessive harvest of medicinal plants in the savannas and forests surrounding large cities. There is thus an urgent need to cultivate medicinal plants in order to conserve biodiversity and protect threatened species (Joshi and Joshi 2014). Population selection in nurseries accompanied by quality control of active ingredient composition could help to identify accessions that can be grown ex situ, thus contributing to the sustainable utilization of medicinal plant species. This is of particular relevance for herbaceous species that are frequently uprooted in the wild, a practice that often results in the extinction of natural populations.

Our study also demonstrates that many wild plants heavily exploited as medicinal remedies in Burkina Faso are not yet finally assessed in their conservation status by IUCN (2018) due to a lack of data. There is an urgent need for assessing the threat status of natural populations of medicinal plants and to strengthen conservation work in order to preserve this cultural heritage for future generations.

\section{Conclusions}

Our study on medicinal plants traded in various regions of Burkina Faso demonstrates that a high diversity of species is used as remedies for many ailments. This complementary medicine is particularly useful for people who cannot afford or do not have access to modern medicinal care. Savanna trees are the most widely used source of medicinal plants. Increased efforts are therefore necessary to preserve the integrity of natural populations of these species regarding their low conservation status. One remedy could be the cultivation of these species in home gardens or nurseries. Our field study could help to focus future conservation efforts and to identify promising candidates for further experimental and clinical research with the objective for developing these plants into standardized drugs.

\section{Declarations}

List of abbreviations: AN: Assemblée Nationale; HNBU: Herbier National du Burkina Faso; IUCN: International Union for Conservation of Nature; MAHRH: Ministère de l'Agriculture de l'Hydraulique et des Ressources Halieutiques; WHO: World Health Organization

Ethics approval and consent to participate: All participants were asked for their free prior informed consent before interviews were conducted.

Consent for publication: Not applicable.

Availability of data and materials: Raw data can be requested from the corresponding author.

Competing interests: The authors declare that they have no competing interests.

Funding: This study has not received any organizational funding.

Authors' contributions: LO designed and performed the field study and together with JE wrote the paper. PAEDS and HS contributed to the drafting of the paper. MK reviewed the final version of the manuscript.

\section{Acknowledgements}

The authors would like to thank the traditional healers in the markets who accepted to provide information for this survey, and the staff of the herbarium "HNBU" for his help in the identification of the samples.

\section{Literature cited}

Agbodeka K, Karou SD, Anani K, Agbonon A. 2016. Ethnobotanical study of medicinal plants used for the treatment of malaria in the Plateau Region, Togo. Pharmacognosy Research 8:10-18.

Ahamidé IDY, Tossou MG, Yédomonhan H, Adomou AC, Houénon J, Akoègnimou A. 2017. Diversité des Loranthaceae et leur impact sur Vitellaria paradoxa C.F. Gaertn: un fruitier à grande valeur socioéconomique au Nord- Bénin. European Scientific Journal 13:217-230.

AN. 1994. Code de la Santé publique au Burkina Faso. pages 39-53. 
AN. 1997. Code forestier au Burkina Faso, pages 78

Andrade-Cetto A, Heinrich M. 2011. From the field into the lab: useful approaches to selecting species based on local knowledge. Frontiers Pharmacology 2:1-5.

Arbonnier M. 2000. Arbres, arbuste et lianes des zones sèches d'Afrique de l'Ouest. CIRAD, Monpellier, MNHN

Benoît E. 2008. Changements climatiques: vulnérabilité, impacts et adaptation dans le monde de la médicine traditionnelle. Vertigo 8: 1-12.

Bhattarai NK. 1997. Biodiversity - people interface in Nepal. In Bodeker G., Bhat K.K.S., Burley J. and Vantomme P. (eds). Medicinal plant for forest conservation and healthcare. Non-wood forest products No. 11, FAO, Rome, Italy.

Cunningham AB. 1997. An Africa-wide overview of medicinal plant harvesting, conservation and health care. In: Bodeker G., Bhat K.K.S., Burley J. and Vantomme P. (eds). Medicinal plant for forest conservation and health care. In: Non-wood forest products No.11. FAO, Rome, Italy.

Cunningham AB. 2014. Applied ethnobotany: people, wild plant use and conservation.1st Edition. WWF, UNESCO, and Royal Botanic Gardens Kew.

Dossou ME, Houessou GL, Lougbégnon OT, Tenté AHB, Codjia JTC. 2012. Etude ethnobotanique des ressources forestières ligneuses de la forêt marécageuse d'Agonvè et terroirs connexes au Bénin. Tropicultura 30:41-48.

FAO. 1996. Burkina Faso: rapport de pays pour la conférence technique internationale de la FAO sur les ressources phytogénétiques. LEIPZIG. 38 pages.

Fontès J, Guinko S. 1995. Carte de la végétation et de l'occupation du sol du Burkina Faso. Notice explicative. Toulouse, France, 67. Pp.

Haidara M, Bourdy G, De Tommasic N, Bracad A, Traore K, Giani S, Sanogo R .2016. Medicinal plants used in Mali for the treatment of malaria and liver diseases. Natural Product Communication 11:339352.

Idu M, Erhabor JO, Efijuemue HM. 2010. Documentation on medicinal plants sold in markets in Abeokuta, Nigeria. Tropical Journal of Pharmaceutical Research 9:110-118.

IUCN. 2018. The IUCN red list of threatened species. [http://www.iucnredlist.org] accessed 19 June 2018.

Iwu MM. 1993. Handbook of African medicinal plants. London: CRC Press Inc.

Joshi BC, Joshi RK. 2014. The role of medicinal plants in livelihood improvement in Uttarakhand. International Journal of Herbal Medicine 1: 55-58

Kasali FM, Mahano AO, Nyakabwa DS, Kadima NJ,
Misakabu FM, Tshibangu DST, Ngbolua KN, Mpiana PT. 2014. Ethnopharmacological survey of medicinal plants used against malaria in Bukavu city (D.R. Congo). European Journal of Medicinal Plants 4:2944.

Konkon NG, Adjoungoua AL, Manda P, Simaga D, N'Guessan KE, Kone BD. 2008. Toxicological and phytochemical screening study of Mitragyna inermis (willd.) O ktze (Rubiaceae), anti diabetic plant. Journal of Medicinal Plants Research 2:279-284.

Kool A, De Boer HJ, Krüger A, Rydberg A, Abbad A, Björk L, Martin G. 2012. Molecular identification of commercialized medicinal plants in Southern Morocco. PLoS One 7:e39459.

Kristensen M, Lykke AM. 2003. Informant-based valuation of use and conservation preferences of savanna trees in Burkina Faso. Economic Botanic 57:203-217.

Ky JMK, Zerbo P, Gnoula C, Simpore J, Nikiema JB, Millogo-Rasolodimby J. 2009. Medicinal plants used in traditional medicine in the Centre East Region of Burkina Faso. Pakistan Journal Biological Sciences 12:1287-1298.

MAHRH. 2007. Rapport national sur l'état des ressources phytogénétiques pour l' alimentation et l'agriculture, Pp. 45.

Maroyi A. 2011. An ethnobotanical survey of medicinal plants used by the people in Nhema communal area, Zimbabwe. Journal of Ethnopharmacology 136:347-354.

Martin GJ. 1995. Ethnobotany. A methods manual. Chapman \& Hall, London, UK.

Moyo M, Aremu AO, Staden JV. 2015. Medicinal plants: an invaluable, dwindling resource in subSaharan Africa. Journal of Ethnopharmacology 174:596-606.

Nadembega P, Issaka J, Baptiste J, Poli F, Antognoni F. 2011. Medicinal plants in Baskoure, Kouritenga Province, Burkina Faso: an ethnobotanical study. Journal of Ethnopharmacology 133:378-395.

Ouédraogo A. 1998. Contribution à l'étude in vivo de l'activité antiplasmodique d'extraits hydroalcooliques de Gardenia sokotensis Hutch (Rubiaceae) chez la souris NMRI infestée par Plasmodium berghei. Thèse de doctorat en pharmacie de l' Université de Ouagadougou.

Senthilkumar K, Aravindhan V, Rajendran A. 2013. Ethnobotanical survey of medicinal plants used by Malayali tribes in Yercaud hills of Eastern Ghats, India. Journal of Natural Remedies 13:119-132.

Sourabie TS, Some N, Bognonou O, Ouattara Y, Ouédraogo JB. 2013. Ethnobotanical and ethnopharmacognostical survey on medicinal plants of Malon village and surrounding in the Cascades 
Region (Burkina Faso). losr Journal of Pharmacy 3:11-15.

Suleiman MHA. 2015. An ethnobotanical survey of medicinal plants used by communities of Northern Kordofan region, Sudan. Journal of Ethnopharmacology 24:232-242.

Tapsoba H, Deschamps J. 2006. Use of medicinal plants for the treatment of oral diseases in Burkina Faso. Journal of Ethnobiology and Ethnomedicine 104:68-78.

Togola A, Diallo D, Dembélé S, Barsett H, Paulsen BS. 2005. Ethnopharmacological survey of different uses of seven medicinal plants from Mali, (West Africa) in the regions Doila, Kolokani and Siby. Journal of Ethnobiology and Ethnomedicine 1:7.

Tugume P, Kakudidi EK, Buyinza M, Namaalwa J, Kamatenesi M, Mucunguzi P, Kaléma J. 2016. Ethnobotanical survey of medicinal plant species used by communities around Mabira Central Forest Reserve, Uganda. Journal of Ethnobiology and Ethnomedicine 12:5.

Uddin ZM, Hassan A. 2014. Determination of informant consensus factor of ethnomedicinal plants used in Kalenga forest, Bangladesh. Bangladesh Journal of Plant Taxonomy 21:83-91.

Van Andel T, Myren B, Van Onselen S. 2012. Ghana's herbal market. Journal of Ethnopharmacology 140:368-378.

WHO. 2004. Guidelines on safety monitoring of herbal medicines in pharmacovigilance systems, Pp. 82.

WHO. 2009. World Malaria Report, Pp. 78.

Willcox M, Benoit-vical F, Fowler D, Bourdy G, Burford G, Giani S, Graziose R, Houghton P, Randrianarivelojosia M, Rasoanaivo P. 2011. Do ethnobotanical and laboratory data predict clinical safety and efficacy of anti-malarial plants? Malaria Journal 10:S7.

Zerbo P, Millogo-Rasolodimby J, NacoulmaOuédraogo OG, Van Damme P. 2011. Plantes médicinales et pratiques médicales au Burkina Faso: cas des Sanan. Bois et Forêts des Tropiques 307:41-53.

Zizka A, Thiombiano A, Dressler S, Nacoulma BMI, Ouédraogo A, Ouédraogo I, Ouédraogo O, Zizka G, Hahn K, Schmidt M. 2015. Traditional plant use in Burkina Faso (West Africa): a national-scale analysis with focus on traditional medicine. Journal of Ethnobiology and Ethnomedicine 11:9. 